\title{
1 Raman2RNA: Live-cell label-free prediction of single-cell RNA 2 expression profiles by Raman microscopy
}

3 Koseki J. Kobayashi-Kirschvink ${ }^{1,2}$, Shreya Gaddam ${ }^{1,9}$, Taylor James-Sorenson ${ }^{1}$, Emanuelle

4 Grody ${ }^{1,3}$, Johain R. Ounadjela ${ }^{1,3}$, Baoliang $\mathrm{Ge}^{4}$, Ke Zhang ${ }^{5}$, Jeon Woong Kang ${ }^{2}$, Ramnik Xavier ${ }^{1,6}$,

5 Peter T. C. So ${ }^{2,4}$, Tommaso Biancalani ${ }^{1,9, \dagger, *}$, Jian Shu ${ }^{1,3,5, \dagger, t}$, Aviv Regev ${ }^{1,7,8,9, \dagger, *}$

$6 \quad{ }^{1}$ Broad Institute of MIT and Harvard, Cambridge, MA 02142, USA

$7 \quad{ }^{2}$ Laser Biomedical Research Center, G. R. Harrison Spectroscopy Laboratory, Massachusetts Institute of

8 Technology, Cambridge, MA 02139, USA

$9 \quad{ }^{3}$ Whitehead Institute for Biomedical Research, Cambridge, MA 02142, USA

$10{ }^{4}$ Department of Mechanical and Biological Engineering, Massachusetts Institute of Technology, Cambridge, MA

1102139, USA

$12{ }^{5}$ Cutaneous Biology Research Center, Massachusetts General Hospital, Harvard Medical School, Boston, MA

13 02129, USA

$14{ }^{6}$ Center for Computational and Integrative Biology and Department of Molecular Biology, Massachusetts General

15 Hospital, Boston, Massachusetts 02114, USA

$16 \quad{ }^{7}$ Department of Biology, Massachusetts Institute of Technology, Cambridge, MA 02139, USA

$17 \quad{ }^{8}$ Howard Hughes Medical Institute, Cambridge, MA 02142, USA

18 9Present address: Genentech, 1 DNA Way, South San Francisco, CA 94080, USA

19 These authors contributed equally

20 †Correspondence: aviv.regev.sc@gmail.com; jian.shu@mgh.harvard.edu; tbiancal@broadinstitute.org 
23 Single cell RNA-Seq (scRNA-seq) and other profiling assays have opened new windows into

24 understanding the properties, regulation, dynamics, and function of cells at unprecedented

25 resolution and scale. However, these assays are inherently destructive, precluding us from

26 tracking the temporal dynamics of live cells, in cell culture or whole organisms. Raman

27 microscopy offers a unique opportunity to comprehensively report on the vibrational energy

28 levels of molecules in a label-free and non-destructive manner at a subcellular spatial

29 resolution, but it lacks in genetic and molecular interpretability. Here, we developed

30 Raman2RNA (R2R), an experimental and computational framework to infer single-cell

31 expression profiles in live cells through label-free hyperspectral Raman microscopy images

32 and multi-modal data integration and domain translation. We used spatially resolved single-

33 molecule RNA-FISH (smFISH) data as anchors to link scRNA-seq profiles to the paired

34 spatial hyperspectral Raman images, and trained machine learning models to infer

35 expression profiles from Raman spectra at the single-cell level. In reprogramming of mouse

36 fibroblasts into induced pluripotent stem cells (iPSCs), R2R accurately ( $>0$ 0.96) inferred

37 from Raman images the expression profiles of various cell states and fates, including iPSCs,

38 mesenchymal-epithelial transition (MET) cells, stromal cells, epithelial cells, and fibroblasts.

39 R2R outperformed inference from brightfield images, showing the importance of

40 spectroscopic content afforded by Raman microscopy. Raman2RNA lays a foundation for

41 future investigations into exploring single-cell genome-wide molecular dynamics through

42 imaging data, in vitro and in vivo.

43 Keywords: Raman microscopy, single-cell transcriptomics, multi-domain translation 
Main

46 Cellular states and functions are determined by a dynamic balance between intrinsic and extrinsic

47 programs. Dynamic processes such as cell growth, stress responses, differentiation, and

48 reprogramming are not determined by a single gene, but by the orchestrated temporal expression

49 and function of multiple genes organized in programs and their interactions with other cells and

50 the surrounding environment ${ }^{1}$. To understand how cells change their states in physiological and

51 pathological conditions it is essential to decipher the dynamics of the underlying gene programs.

52 Despite major advances in single cell genomics and microscopy, we still cannot track live cells

53 and tissues at the genomic level. On the one hand, single cell and spatial genomics have provided

54 a view of gene programs and cell states at unprecedented scale and resolution ${ }^{1}$, but these

55 measurement methods are destructive, and involve tissue fixation and freezing and/or cell lysis,

56 precluding us from directly tracking the dynamics of full molecular profiles in live cells or

57 organisms. While advanced computational methods, such as pseudo-time algorithms (e.g.,

58 Monocle $^{2}$, Waddington-OT $\left.{ }^{3}\right)$ and velocity-based methods (e.g., velocyto ${ }^{4}$, scVelo $^{5}$ ), can infer

59 dynamics from snapshots of molecular profiles, they rely on assumptions that remain challenging

60 to verify experimentally ${ }^{6}$. On the other hand, fluorescent reporters can be used to monitor the

61 dynamics of individual genes and programs within live cells, but are limited in the number of

62 targets they can report ${ }^{7}$, must be chosen ahead of the experiment and often involve genetically

63 engineered cells. Moreover, the vast majority of dyes and reporters require fixation or can interfere

64 with nascent biochemical processes and alter the natural state of the gene of interest ${ }^{7}$. Therefore,

65 it remains technically challenging to dynamically monitor the activity of a large number of genes

66 simultaneously. 
67 Raman microscopy opens a unique opportunity for monitoring live cells and tissues, as it

68 collectively reports on the vibrational energy levels of molecules in a label-free and non-

69 destructive manner at a subcellular spatial resolution, thus providing molecular fingerprints of

70 cells $^{8}$. Pioneering research has demonstrated that Raman microscopy can be used for

71 characterizing cell types and cell states $^{8}$, non-destructively diagnosing pathological specimens

72 such as tumors ${ }^{9}$, characterizing the developmental states of embryos ${ }^{10}$, and identifying bacteria

73 with antibiotic resistance ${ }^{11}$. However, the complex and high-dimensional nature of the spectra, the

74 spectral overlaps of biomolecules such as proteins and nucleic acids, and the lack of unified

75 computational frameworks have hindered the decomposition of the underlying molecular

76 profiles $^{7,8}$.

77 To address this challenge and leverage the complementary strengths of Raman microscopy and

78 scRNA-Seq, we developed Raman2RNA (R2R), an experimental and computational framework

79 for inferring single-cell RNA expression profiles from label-free non-destructive Raman

80 hyperspectral images (Fig. 1). R2R takes as input spatially resolved hyperspectral Raman images

81 from live cells, smFISH data of selected markers from the same cells, and scRNA-seq from the

82 same biological system. R2R then uses the smFISH data as an anchor to learn a model that links

83 spatially resolved hyperspectral Raman images to scRNA-seq. Finally, from this model, R2R then

84 computationally infers the anchor smFISH measurements from hyperspectral Raman images and

85 then the single-cell expression profiles. The result is a label-free live-cell inference of single-cell

86 expression profiles.

87 To facilitate data acquisition, we developed a high-throughput multi-modal spontaneous Raman

88 microscope that enables automated acquisition of Raman spectra, brightfield, and fluorescent

89 images. In particular, we integrated Raman microscopy optics to a fluorescence microscope, where 
90 high-speed galvo mirrors and motorized stages were combined to achieve a large field of view

91 (FOV) scanning, and where dedicated electronics automate measurements across multiple

92 modalities (Extended Data Fig. 1-2, Methods).

93 We first demonstrated that R2R can infer profiles of two distinct cell types: mouse induced

94 pluripotent stem cells (iPSCs) expressing an endogenous Oct4-GFP reporter and mouse

95 fibroblasts ${ }^{12}$. To this end, we mixed the cells in equal proportions, plated them in a gelatin-coated

96 quartz glass-bottom Petri dish, and performed live-cell Raman imaging, along with fluorescent

97 imaging of live-cell nucleus staining dye (Hoechst 33342) for cell segmentation and image

98 registration, and an iPSC marker gene, Oct4-GFP (Fig. 2a). The excitation wavelength for our

99 Raman microscope $(785 \mathrm{~nm})$ was distant enough from the GFP Stokes shift emission, such that

100 there was no interference with the cellular Raman spectra (Extended Data Fig. 3). Furthermore,

101 there was no notable photo-toxicity induced in the cells. After Raman and fluorescence imaging,

102 we fixed and permeabilized the cells and performed smFISH (with hybridization chain reaction

103 (HCR $\left.{ }^{13}\right)$, Methods) of marker genes for mouse iPSCs (Nanog) and fibroblasts (Collal). We

104 registered the nuclei stains, GFP images, HCR images, and Raman images through either

105 polystyrene control bead images or reference points marked under the glass bottom dishes

106 (Extended Data Fig. 4, Methods).

107 The Raman spectra distinguished the two cell populations in a manner congruent with the

108 expression of their respective reporter (measured live or by smFISH in the same cells), as reflected

109 by a low-dimensional embedding of hyperspectral Raman data (Fig. 2b). Specifically, we focused

110 on the fingerprint region of Raman spectra $\left(600-1800 \mathrm{~cm}^{-1}, 930\right.$ of the 1,340 features in a Raman

111 spectrum), where most of the signatures from various key biomolecules, such as proteins, nucleic

112 acids, and metabolites, lie $^{8}$. After basic preprocessing, including cosmic-ray and background 
113 removal and normalization, we aggregated Raman spectra that are confined to the nuclei, obtaining

114 a 930-dimensional Raman spectroscopic representation for each cell's nucleus. We then visualized

115 these Raman profiles in an embedding in two dimensions using Uniform Manifold Approximation

116 and Projection (UMAP) ${ }^{14}$ and labeled cells with the gene expression levels that were concurrently

117 measured by either an Oct4-GFP reporter or smFISH (Fig. 2b). The cells separated clearly in their

118 Raman profiles in a manner consistent with their gene expression characteristics, forming two

119 main subsets in the embedding, one with cells with high Oct4 and Nanog expression (iPSCs

120 markers) and another with cells with relatively high Collal expression (fibroblasts marker),

121 indicating that Raman spectra reflect cell-intrinsic expression differences (Fig. 2b).

122 We further successfully trained a classifier to classify the 'on' or 'off' expression states of Oct4,

123 Nanog and Collal in each cell based on its Raman profile (Methods). We trained a logistic

124 regression classifier with $50 \%$ of the data and held out $50 \%$ for testing. We predicted Oct4 and

125 Nanog expression states with high accuracy on the held-out test data (area under the receiver

126 operating characteristic curve $($ AUROC $)=0.98$ and 0.95 , respectively; Fig. 2c), indicating that

127 expression of iPSC markers can be predicted confidently from Raman spectra of live, label-free

128 cells. We also successfully classified the expression state of the fibroblast marker Collal

129 (AUROC = 0.87; Fig. 2c), albeit with lower confidence, which is consistent with the lower contrast

130 in Collal expression (Fig. 2b) between iPSC (Oct4+ or Nanog+ cells) vs. non-iPSCs, compared

131 to Oct4 or Nanog. Most misclassifications occurred when the ground truth expression levels were

132 near the threshold of the classifier, showing that misclassifications were likely due to the

133 uncertainty in the ground truth expression level (Extended Data Fig. 5).

134 Next, we asked if the Raman images could predict entire expression profiles non-destructively at 135 single-cell resolution. To this end, we aimed to reconstruct scRNA-seq profiles from Raman 
images by multi-modal data integration and translation, using multiplex smFISH data to anchor between the Raman images and scRNA-seq profiles (Fig. 3a). As a test case, we focused on the mouse iPSC reprogramming model system, where we have previously generated $\sim 250,000$ 139 scRNA-seq profiles at $1 / 2$ day intervals throughout an 18 day, 36 time point time course of 140 reprogramming ${ }^{3}$ (Methods). We used Waddington-OT ${ }^{3}$ (WOT) to select from the scRNA-seq 141 profiles nine anchor genes that represent diverse cell types that emerge during reprogramming 142 (iPSCs: Nanog, Utf1 and Epcam; MET and neural: Nnat and Fabp7; epithelial: Krt7 and Peg10;

143 stromal: Bgn and Colla1; Methods). We performed live-cell Raman imaging from day 8 of 144 reprogramming, in which distinct cell types begin to emerge ${ }^{3}$, up to day 14.5 , at half-day intervals, 145 totaling 14 time points (Methods). We imaged $\sim 500$ cells per plate at $1 \mu \mathrm{m}$ spatial resolution.

146 Finally, we fixed cells immediately after each Raman imaging time point followed by smFISH on 147 the 9 anchor genes (Methods).

148 Strikingly, a low dimensional representation of the Raman profiles showed that they encoded 149 similar temporal dynamics to those observed with scRNA-seq during reprogramming (Fig. 3b,c, 150 Extended Data Fig. 6), indicating that they may qualitatively mirror scRNA-seq.

151 Integrating Raman and scRNA-seq profiles (Methods), R2R then learned a model that can infer 152 an scRNA-seq profile for each Raman imaged cell, by first predicting smFISH anchors from the 153 Raman profiles using Catboost ${ }^{15}$ (Methods) and then using our Tangram ${ }^{16}$ method to map from 154 the anchors to full scRNA-seq profiles (Fig. 1, Fig. 3d-f). In the first step, we averaged the smFISH 155 signal within a nucleus to represent a single nucleus's expression level. As we conducted smFISH 156 of 9 genes, the result was a 9-dimensional smFISH profile for each single nucleus. Then, Raman 157 profiles were translated to these 9-dimensional profiles with Catboost ${ }^{15}$, a non-linear regression 158 model, using $50 \%$ of the Raman and smFISH profiles as training data. 
In the second step, we mapped these anchor smFISH profiles to full scRNA-seq profiles using Tangram, yielding well-predicted single cell RNA profiles, as supported by several lines of evidence. First, we performed leave-one-out cross-validation (LOOCV) analysis, in which we used eight out of the nine anchor genes to integrate Raman with scRNA-seq, and compared the predicted expression of the remaining genes to its smFISH measurements. The predicted left-out genes based on scRNA-seq showed a significant correlation with the measured smFISH expression for any leftout gene (Pearson $r \sim 0.7, p$-value $<10^{-100}$, Fig. 3d). Notably, when we analogously applied a modified U-net ${ }^{18}$ to infer smFISH profiles from brightfield (Extended Data Fig. 15, Methods), we observed a poor, near-random prediction of expression profiles for all 9 genes in leave-one-out cross-validation $(r<0.15)$, indicating that, unlike Raman spectra, brightfield z-stack images either do not have the necessary information to infer expression profiles, or require more data. Second, we compared the real (scRNA-seq measured) and R2R predicted expression profiles averaged

171 across cells of the same cell type ("pseudobulk" for each of iPSCs, epithelial cells, stromal cells, and MET). Here, we obtained the "ground truth" cell types of the R2R profiles by transferring

173 scRNA-seq annotations to the matching smFISH profiles using Tangram's label transfer function.

174 Then, based on the labels, we averaged R2R's predicted profiles across the cells of a single cell

175 type. The two profiles (R2R-inferred and scRNA-seq pseudo-bulk per cell type) showed high 176 correlations (Pearson's $r>0.96$ ) (Fig. 3e,f, Extended Data Fig. 7), demonstrating the accuracy of 177 R2R at the cell type level. Furthermore, projecting the R2R predicted profiles of each cell onto an 178 embedding learned from the real scRNA-seq shows that the predicted profiles span the key cell 179 types as captured in real profiles (Fig. 3g-j, Extended Data Fig. 8-12). We note that the predicted profiles had lower variance compared to real scRNA-seq. As this is observed even when co181 embedding only smFISH and scRNA-seq measurements (with no Raman data or projection, 
182 Extended Data Fig. 13), we believe it mostly reflects the limited number and domain

183 maladaptation of the smFISH anchor genes used for integration. Given the similarity of the

184 separate embeddings of Raman and scRNA-seq profiles, future studies without anchors could

185 address this.

186 Lastly, we calculated feature importance scores in R2R predictions (Methods) and identified

187 Raman spectral features correlated with expression levels (Fig. 3k, Extended Data Fig. 14). For

188 example, Raman bands at approximately $752 \mathrm{~cm}^{-1}$ (C-C, Try, cytochrome), $1004 \mathrm{~cm}^{-1}$ (CC, Phe,

189 Tyr), and $1445 \mathrm{~cm}^{-1}\left(\mathrm{CH}_{2}\right.$, lipids) contributed to predicting iPSCs-related expression profiles,

190 which is consistent with previous research that employed single cell Raman spectra to identify

191 mouse embryonic stem cells $(\mathrm{ESCs})^{17}$ (Fig. 3k). The contributions of these bands were either

192 suppressed or increased for other cell types, such as stromal or epithelial cells (Extended Data

193 Fig. 14).

194 In conclusion, we reported R2R, a label-free non-destructive framework for inferring expression 195 profiles at single-cell resolution from Raman spectra of live cells, by integrating Raman 196 hyperspectral images with scRNA-seq data through paired smFISH measurements and multi197 modal data integration and translation. We inferred single-cell expression profiles with high 198 accuracy, based on both averages within cell types and co-embeddings of individual profiles. We 199 further showed that predictions using brightfield z-stacks had poor performance, indicating the 200 importance of Raman microscopy for predicting expression profiles.

201 R2R can be further developed in several ways. First, the throughput of single-cell Raman 202 microscopy is still limited. In this pilot study, we profiled $\sim 6,000$ cells in total. By using emerging 203 vibrational spectroscopy techniques, such as Stimulated Raman Scattering microscopy ${ }^{19}$ or photo- 
204 thermal microscopy ${ }^{20,21}$, we envision increasing throughput by several orders of magnitude, to

205 match the throughput of massively parallel single cell genomics. Second, because molecular

206 circuits and gene regulation are structured, with strong co-variation in gene expression profiles

207 across cells, we can leverage the advances in computational microscopy to infer high-resolution

208 data from low-resolution data, such as by using compressed sensing, to further increase

209 throughput ${ }^{22}$. Third, increasing the number of anchor genes (e.g., by seqFISH ${ }^{23}$, merFISH $^{24}$,

210 STARmap $^{25}$, or ExSeq ${ }^{26}$ ) can increase our prediction accuracy and capture more single-cell

211 variance. Additionally, with single-cell multi-omics, we can project other modalities, such as

212 scATAC-seq from Raman spectra. Finally, given the similarity in the overall independent

213 embedding of Raman and scRNA-seq profiles, we expect computational methods such as multi-

214 domain translation ${ }^{27}$ to allow mapping between Raman spectra and molecular profiles without

215 measuring any anchors in situ. Overall, with further advances in single-cell genomics, imaging,

216 and machine learning, Raman2RNA could allow us to non-destructively infer omics profiles at

217 scale in vitro, and possibly in vivo in living organisms. 
Materials and Methods

Mouse fibroblast reprogramming

221 OKSM secondary mouse embryonic fibroblasts (MEFs) were derived from E13.5 female embryos

222 with a mixed B6;129 background. The cell line used in this study was homozygous for ROSA26-

223 M2rtTA, homozygous for a polycistronic cassette carrying Oct4, Klf4, Sox2, and Myc at the

224 Collal 3' end, and homozygous for an EGFP reporter under the control of the Oct4 promoter.

225 Briefly, MEFs were isolated from E13.5 embryos from timed-matings by removing the head,

226 limbs, and internal organs under a dissecting microscope. The remaining tissue was finely minced

227 using scalpels and dissociated by incubation at $37^{\circ} \mathrm{C}$ for 10 minutes in trypsin-EDTA

228 (ThermoFisher Scientific). Dissociated cells were then plated in MEF medium containing DMEM

229 (ThermoFisher Scientific), supplemented with 10\% fetal bovine serum (GE Healthcare Life

230 Sciences), non-essential amino acids (ThermoFisher Scientific), and GlutaMAX (ThermoFisher

231 Scientific). MEFs were cultured at $37^{\circ} \mathrm{C}$ and $4 \% \mathrm{CO}_{2}$ and passaged until confluent. All procedures,

232 including maintenance of animals, were performed according to a mouse protocol (2006N000104)

233 approved by the MGH Subcommittee on Research Animal Care ${ }^{3}$.

234 For the reprogramming assay, 50,000 low passage MEFs (no greater than 3-4 passages from 235 isolation) were seeded in $143.5 \mathrm{~cm}$ quartz glass-bottom Petri dishes (Waken B Tech) coated with

236 gelatin. These cells were cultured at $37^{\circ} \mathrm{C}$ and $5 \% \mathrm{CO}_{2}$ in reprogramming medium containing

237 KnockOut DMEM (GIBCO), 10\% knockout serum replacement (KSR, GIBCO), 10\% fetal bovine

238 serum (FBS, GIBCO), 1\% GlutaMAX (Invitrogen), 1\% nonessential amino acids (NEAA, 239 Invitrogen), $0.055 \mathrm{mM}$ 2-mercaptoethanol (Sigma), 1\% penicillin-streptomycin (Invitrogen) and $2401,000 \mathrm{U} / \mathrm{ml}$ leukemia inhibitory factor (LIF, Millipore). Day 0 medium was supplemented with 2 
$241 \mathrm{mg} / \mathrm{mL}$ doxycycline Phase-1 (Dox) to induce the polycistronic OKSM expression cassette. The

242 medium was refreshed every other day. On day 8, doxycycline was withdrawn. Fresh medium was

243 added every other day until the final time point on day 14 . One plate was taken every 0.5 days

244 after day 8 (D8-D14.5) for Raman imaging and fixed with 4\% formaldehyde immediately after for 245 HCR.

\section{High-throughput multi-modal Raman microscope}

247 Due to the lack of commercial systems, we developed an automated high-throughput multi-modal 248 microscope capable of multi-position and multi-timepoint fluorescence imaging and point 249 scanning Raman microscopy (Extended Data Fig. 1). A 749 nm short-pass filter was placed to 250 separate brightfield and fluorescence from Raman scattering signal, and the fluorescence and

251 Raman imaging modes were switched by swapping dichroic filters with auto-turrets. To realize a

252 high-throughput Raman measurement, galvo mirror-based point scanning and stage scanning was 253 combined to acquire each FOV and multiple different FOVs, respectively.

254 To realize this in an automated fashion, a MATLAB (2020b) script that communicates with Micro255 manager ${ }^{28}$, a digital acquisition (DAQ) board, and Raman scattering detector (Princeton 256 Instruments, PIXIS 100BR eXcelon) was written (Extended Data Fig. 2). A 2D point scan Raman

257 imaging sequence was regarded as a dummy image acquisition in Micro-manager, during which

258 the script communicated via the DAQ board with 1. the detector to read out a spectrum, 2. the 259 mirror to update the mirror angles, and 3. shutters to control laser exposure. All communications 260 were realized using transistor-transistor logic (TTL) signaling. Updating of the galvo mirror angles 261 was conducted during the readout of the detector. While the script ran in the background, Micro- 
manager initiated a multi-dimensional acquisition consisting of brightfield, DAPI, GFP, and dummy Raman channel at multiple positions and z-stacks.

264 An Olympus IX83 fluorescence microscope body was integrated with a $785 \mathrm{~nm}$ Raman excitation

265 laser coupled to the backport, where the short-pass filter deflected the excitation to the sample 266 through an Olympus UPLSAPO 60X NA 1.2 water immersion objective. The backscattered light

267 was collimated through the same objective and collected with a $50 \mu \mathrm{m}$ core multi-mode fiber, 268 which was then sent to the spectrograph (Holospec f/1.8i $785 \mathrm{~nm}$ model) and detector. The 269 fluorescence and brightfield channels were imaged by the Orca Flash $4.0 \mathrm{v} 2 \mathrm{sCMOS}$ camera from 270 Hamamatsu Photonics. The exposure time for each point in the Raman measurement was $20 \mathrm{msec}$, 271 and laser power at the sample plane was $212 \mathrm{~mW}$. Each FOV was 100x100 pixels, with each pixel 272 corresponding to about $1 \mu \mathrm{m}$. The laser source was a $785 \mathrm{~nm}$ Ti-Sapphire laser cavity coupled to 273 a $532 \mathrm{~nm}$ pump laser operating at $4.7 \mathrm{~W}$.

274 The time to acquire Raman hyperspectral images was roughly 8 minutes per FOV. With 8 minutes, 275 it is unrealistic to image an entire glass-bottom plate. Therefore, we visually chose representative 276 FOVs that cover all representative cell types including iPSC-like, epithelial-like, stromal-like and 277 MET cells. 20 FOVs were chosen for each plate, where roughly 15 FOVs were from the boundaries 278 of colonies, five from non-colonies, and one from non-cells to use for background correction.

279 Due to the extended Raman imaging time, evaporation of the immersion water was no longer 280 negligible. Therefore, we developed an automated water immersion feeder using syringe pumps 281 and syringe needles glued to the tip of the objective lens. Here, water was supplied at a flow rate 282 of $1 \mu \mathrm{L} / \mathrm{min}$. 


\section{iPSC and MEF mixture experiment}

284 Low passage iPSCs were first cultured in N2B27 2i media containing 3 mM CHIR99021, $1 \mathrm{mM}$

285 PD0325901, and LIF. On the day of the experiment, 750,000 iPSCs and 750,000 MEFs were plated

286 on the same gelatin-coated $3.5 \mathrm{~cm}$ quartz glass-bottom Petri dish. Cells were plated in the same

287 reprogramming medium as previously described (with Dox) with the exception of utilizing DMEM

288 without phenol red (Gibco) instead of KnockOut DMEM. 6 hours after plating, the quartz dishes

289 were taken for Raman imaging and fixed with 4\% formaldehyde immediately after for HCR.

\section{Anchor gene selection by Waddington-OT}

291 To select anchor genes for connecting spatial information to the full transcriptome data,

292 Waddington-OT $(\text { WOT })^{3}$, a probabilistic time-lapse algorithm that can reconstruct developmental

293 trajectories, was used. We applied WOT to mouse fibroblast reprogramming scRNA-seq data

294 collected at matching time-points and culture condition (day 8-14.5 at $1 / 2$ day intervals) ${ }^{3}$. For each

295 cell fate, we calculated the transition probabilities of each cell and selected the top 10 percentile

296 cells per time point (Extended Data Fig. 6). Based on this, we ran the FindMarker function in

297 Seurat $^{29}$ to find genes differentially expressed in these cell subsets per time point. Through this

298 approach, we chose two genes per cell type that are both found by Seurat and commonly used for

299 these cell types (iPSCs: Nanog, Utf1; epithelial: Krt7, Peg10; stromal: Bgn, Collal; MET and

300 neural: Fabp7, Nnat), along with one gene that is an early marker of iPSCs, Epcam.

302 Fixed samples were prepared for imaging using the HCR v3.0 protocol for mammalian cells on a

303 chambered slide, incubating at the amplification step for 45 minutes in the dark at room 
304 temperature. Three probes with amplifiers conjugated to fluorophores Alexa Fluor 488, Alexa

305 Fluor 546, and Alexa Fluor 647 were used. Samples were stained with DAPI prior to imaging.

306 After imaging, probes were stripped from samples by washing samples once for 5 minutes in $80 \%$

307 formamide at room temperature and then incubating three times for 30 minutes in $80 \%$ formamide

308 at $37^{\circ} \mathrm{C}$. Samples were washed once more with $80 \%$ formamide, then once with PBS, and reprobed

309 with another panel of probes for subsequent imaging.

\section{Image registration of Raman hyperspectral images and fluorescence/smFISH images}

311 Brightfield and fluorescence channels including DAPI and GFP, along with corresponding Raman

312 images, were registered by using $5 \mu \mathrm{m}$ polystyrene beads deposited on quartz glass-bottom Petri

313 dishes (SF-S-D12, Waken B Tech) for calibration. The brightfield and fluorescence images of the

314 beads were then registered by the scale-invariant template matching algorithm of the OpenCV

315 (https://github.com/opencv/opencv) matchTemplate function followed by manual correction.

316 For the registration of smFISH and Raman images, four marks inscribed under the glass-bottom

317 Petri dishes were used as reference points (Extended Data Fig. 4). As the Petri dishes are

318 temporarily removed from the Raman microscope after imaging to do smFISH measurements, the

319 dishes cannot be placed back at the same exact location on the microscope. Therefore, the

320 coordinates of these reference points were measured along with the different FOVs. When the

321 dishes were placed again after smFISH measurements, the reference mark coordinates were

322 measured, and an affine mapping was constructed to calculate the new FOV coordinates. Lastly,

323 as smFISH consisted of 3 rounds of hybridization and imaging, the following steps were performed

324 to register images across different rounds with a custom MATLAB script:

325 1. Maximum intensity projection of nuclei stain and RNA images 
2. Automatic registration of round 1 images to rounds 2 and 3 based on nuclei stain images and MATLAB function imregtform. First, initial registration transformation functions were obtained with a similarity transformation model passing the 'multimodal' configuration. Then, those transformations were used as the initial conditions for an affine model-based registration with the imregtform function. Finally, this affine mapping transformation was applied to all the smFISH (RNA) images.

3. Use the protocol in (2) to register nuclei stain images obtained from the multimodal Raman microscope and the $1^{\text {st }}$ round of images used for smFISH. Then, apply the transformation to the remaining $2^{\text {nd }}$ and $3^{\text {rd }}$ rounds.

4. Manually remove registration outliers in (3).

336 Fibroblast cells were mobile during the 2-class mixture experiment so that by the time Raman 337 imaging finished, cells had moved far enough from their original position that the above semi338 automated strategy could not be applied. Thus, we manually identified cells present in both nuclei

339 stain images before and after the Raman imaging.

\section{Hyperspectral Raman image processing}

341 Each raw Raman spectrum has 1,340 channels. Of those channels, we extracted the fingerprint

342 region $\left(600-1800 \mathrm{~cm}^{-1}\right)$, which resulted in a total of 930 channels per spectrum. Thus, each FOV 343 is a 100x100x930 hyperspectral image. The hyperspectral images were then preprocessed by a

344 python script as follows: 
1. Cosmic ray removal. Cosmic rays were detected by subtracting the median filtered spectra from the raw spectra, and any feature above 5 was classified as an outlier and replaced with the median value. The kernel window size for the median filter was 7 . (https://github.com/charlesll/rampy), a python package for Raman spectral preprocessing, was used with the alternating least squares algorithm 'als'.

3. Savitzky-Golay smoothing. The scipy.signal.savgol_filter function was used with window size 5 and polynomial order 3.

4. Averaging spectra at the single-cell level. Nuclei stain images were segmented using NucleAIzer (https://github.com/spreka/biomagdsb) and averaged pixel-level spectra that fall within each nucleus.

5. Spectra standardization. Spectra were standardized to a mean of 0 and a standard deviation of 1 .

\section{Inferring anchor smFISH from Raman spectra or brightfield z-stacks}

359 For the two-class mixture and reprogramming experiment, we trained a decision tree-based nonlinear regression, Catboost ${ }^{15}$, to predict the 'on' or 'off' expression states for each anchor gene

361 from Raman spectra. We used $80 \%$ of the data as training and the remaining $20 \%$ as test data. The 362 early stopping parameter was set to 5.

363 For the brightfield z-stack to smFISH inference, we applied deep learning to the whole image level.

364 We trained a modified U-net with skip connections and residual blocks to estimate the 365 corresponding smFISH image ${ }^{18}$. Due to the small size of the available training dataset, we 366 augmented the data by rotation and flipping. Furthermore, a subsample of each brightfield image 
was taken due to memory constraints (50x50 pixel region). Training was carried out on an NVIDIA

368 Tesla P100 GPU, the number of epochs was 100, the learning rate was 0.01 , and the batch size was 400 . For each smFISH prediction, we chose the epoch that gave the best validation score.

\section{Inferring expression profiles from Raman images}

371 To infer expression profiles from Raman images, we used Tangram ${ }^{16}$. Tangram enables the

372 alignment of spatial measurements of a small number of genes to scRNA-seq measurements. After

373 using Catboost to infer anchor expression levels from Raman profiles, we aligned the inferred

374 expression levels to scRNA-seq profiles using the map_cells_to_space function

375 (learning_rate $=0.1$, num_epochs $=1000$ ) on an Nvidia Tesla P100 GPU, followed by the 376 project_genes function in Tangram.

377 When comparing different pseudo-bulk transcriptome predictions with the real scRNA-seq data, 378 we first transferred labels of annotated scRNA-seq profiles to the ground truth smFISH profiles 379 using Tangram's label transfer function project_cell_annotations. Then, the average expression 380 profiles across cells of a cell type were calculated by referring to the transferred labels and 381 compared with those from the real scRNA-seq data ${ }^{3}$.

\section{Dimensionality reduction, embedding and projection}

383 For dimension reduction and visualization of Raman and scRNA-seq profiles, we performed

384 forced layout embedding (FLE) using the Pegasus pipeline (https://github.com/klarman-cell385 observatory/pegasus). First, we performed principal component analysis on both Raman and 386 scRNA-seq profiles independently, calculated diffusion maps on the top 100 principal 387 components, and performed an approximated FLE graph using Deep Learning by pegasus.net_fle 388 with default parameters. 
To project Raman profiles to a scRNA-seq embedding, we calculated a k-nearest neighbor graph $(k$-NN, $k=15)$ on the scRNA-seq top 50 principal components with the cosine metric, and UMAP with the scanpy.tl.umap function in $\mathrm{Scanpy}^{30}$ version 1.7.2 with default parameters. Then, the Raman predicted expression profiles were projected on to the scRNA-seq UMAP embedding by scanpy.tl.ingest using $\mathrm{k}-\mathrm{NN}$ as the labeling method and default parameters.

\section{$394 \quad$ Feature importance analysis}

395 To evaluate the contributions of Raman spectral features to expression profile prediction, we used 396 the get_feature_importance function in Catboost with default parameters. As the dimensions of 397 Raman spectra were reduced by PCA prior to Catboost, feature importance scores were calculated 398 for each principal component, and the weighted linear combination of the Raman PCA eigen 399 vectors with feature scores as the weight were calculated to obtain the full spectrum.

\section{Author contributions}

401 KJKK, JS, TB and AR conceived the research and developed the methodology. JS, TB and AR

402 funded and supervised research. KJKK, JS, JO performed reprogramming experiments. KJKK

403 developed the multi-modal Raman microscope and control software with supervision from JWK

404 and PS. KJKK, EG, and KZ performed smFISH. KJKK, SG, TJS, and TB developed the Raman

405 spectral preprocessing and classification pipeline. KJKK developed the image registration

406 pipeline, and performed Waddington-OT, Tangram and feature importance analysis. KJKK and

407 BG performed U-net. KJKK, JS, and AR wrote the manuscript with input from all the authors. 


\section{Competing interests statement}

409 AR is a co-founder and equity holder of Celsius Therapeutics, an equity holder in Immunitas, and

410 was a scientific advisory board member of ThermoFisher Scientific, Syros Pharmaceuticals,

411 Neogene Therapeutics and Asimov until 31 July 2020. AR, TB, and SG are employees of

412 Genentech from August 1, 2020, respectively. A patent application has been filed related to this

413 work.

\section{Acknowledgements}

415 KJKK was supported by the Japan Society for the Promotion of Science Postdoctoral Fellowship

416 for Overseas Researchers, and the Naito Foundation Overseas Postdoctoral Fellowship. BG was

417 supported by the MathWorks Fellowship. JS was supported by the Helen Hay Whitney Foundation

418 and NIH Pathway to Independence Award (1K99HD096049-01, 5K99HD096049-02,

419 4R00HD096049-03), and funds from the Broad Institute of MIT and Harvard and Massachusetts

420 General Hospital. This research was funded by NIH National Institute of Biomedical Imaging and

421 Bioengineering, grant P41EB015871 (JWK, PS), NIH grant U19 MH114821 (TB), HubMap

422 UH3CA246632 (TB), and HHMI and the Klarman Cell Observatory (AR). AR was a Howard

423 Hughes Medical Institute Investigator when this work was initiated. We thank Eric Lander, Rudolf

424 Jaenisch, Doeke Hekstra, Joseph Kirschvink for their helpful discussion and insights. We thank 425 Leslie Gaffney for creating and editing figures. 


\section{References}

428 1. Tanay, A. \& Regev, A. Scaling single-cell genomics from phenomenology to mechanism. Nature 541, 331-338 (2017).

2. Trapnell, C. et al. The dynamics and regulators of cell fate decisions are revealed by pseudotemporal ordering of single cells. Nat. Biotechnol. 32, 381-386 (2014).

3. Schiebinger, G. et al. Optimal-Transport Analysis of Single-Cell Gene Expression Identifies Developmental Trajectories in Reprogramming. Cell 176, 928-943.e22 (2019).

4. La Manno, G. et al. RNA velocity of single cells. Nature 560, 494-498 (2018).

5. Bergen, V., Lange, M., Peidli, S., Wolf, F. A. \& Theis, F. J. Generalizing RNA velocity to transient cell states through dynamical modeling. Nat. Biotechnol. 38, 1408-1414 (2020).

6. Wagner, D. E. \& Klein, A. M. Lineage tracing meets single-cell omics: opportunities and challenges. Nat. Rev. Genet. 21, 410-427 (2020).

439 7. Wei, L. et al. Super-multiplex vibrational imaging. Nature 544, 465-470 (2017).

440 8. Kobayashi-Kirschvink, K. J. et al. Linear Regression Links Transcriptomic Data and $441 \quad$ Cellular Raman Spectra. Cell Systems vol. 7 104-117.e4 (2018).

442 9. Singh, S. P. et al. Label-free characterization of ultra violet-radiation-induced changes in skin fibroblasts with Raman spectroscopy and quantitative phase microscopy. Sci. Rep. 7,

10. Ichimura, T. et al. Visualizing cell state transition using Raman spectroscopy. PLoS One 9, $446 \quad$ e84478 (2014).

447 11. Ho, C.-S. et al. Rapid identification of pathogenic bacteria using Raman spectroscopy and deep learning. Nat. Commun. 10, 4927 (2019).

449 12. Stadtfeld, M., Maherali, N., Borkent, M. \& Hochedlinger, K. A reprogrammable mouse strain from gene-targeted embryonic stem cells. Nat. Methods 7, 53-55 (2010). 
451 13. Choi, H. M. T. et al. Third-generation in situ hybridization chain reaction: multiplexed,

452 quantitative, sensitive, versatile, robust. Development 145, (2018).

453 14. McInnes, L., Healy, J., Saul, N. \& Großberger, L. UMAP: Uniform Manifold

454 Approximation and Projection. J. Open Source Softw. 3, 861 (2018).

455 15. Prokhorenkova, L., Gusev, G., Vorobev, A., Dorogush, A. V. \& Gulin, A. CatBoost:

456 unbiased boosting with categorical features.

457 16. Biancalani, T. et al. Deep learning and alignment of spatially-resolved whole transcriptomes

458 of single cells in the mouse brain with Tangram. bioRxiv 2020.08.29.272831 (2020)

459 doi:10.1101/2020.08.29.272831.

460 17. Germond, A., Panina, Y., Shiga, M., Niioka, H. \& Watanabe, T. M. Following Embryonic

$461 \quad$ Stem Cells, Their Differentiated Progeny, and Cell-State Changes During iPS

462 Reprogramming by Raman Spectroscopy. Anal. Chem. 92, 14915-14923 (2020).

463 18. He, K., Zhang, X., Ren, S. \& Sun, J. Deep residual learning for image recognition. in 2016

464 IEEE Conference on Computer Vision and Pattern Recognition (CVPR) (IEEE, 2016).

465 doi:10.1109/cvpr.2016.90.

466 19. Freudiger, C. W. et al. Label-free biomedical imaging with high sensitivity by stimulated

467 Raman scattering microscopy. Science 322, 1857-1861 (2008).

468 20. Bai, Y. et al. Ultrafast chemical imaging by widefield photothermal sensing of infrared $469 \quad$ absorption. Sci Adv 5, eaav7127 (2019).

470 21. Tamamitsu, M., Toda, K., Horisaki, R. \& Ideguchi, T. Quantitative phase imaging with 471 molecular vibrational sensitivity. Opt. Lett. 44, 3729-3732 (2019). 
472 22. Cleary, B., Cong, L., Cheung, A., Lander, E. S. \& Regev, A. Efficient Generation of

473 Transcriptomic Profiles by Random Composite Measurements. Cell 171, 1424-1436.e18

474 (2017).

475 23. Eng, C.-H. L. et al. Transcriptome-scale super-resolved imaging in tissues by RNA

$476 \quad$ seqFISH. Nature 568, 235-239 (2019).

477 24. Chen, K. H., Boettiger, A. N., Moffitt, J. R., Wang, S. \& Zhuang, X. RNA imaging.

478 Spatially resolved, highly multiplexed RNA profiling in single cells. Science 348, aaa6090

$479 \quad(2015)$

480 25. Wang, X. et al. Three-dimensional intact-tissue sequencing of single-cell transcriptional states. Science (2018) doi:10.1126/science.aat5691.

482 26. Alon, S. et al. Expansion sequencing: Spatially precise in situ transcriptomics in intact 483 biological systems. Science 371, (2021).

484 27. Yang, K. D. et al. Multi-domain translation between single-cell imaging and sequencing 485 data using autoencoders. Nat. Commun. 12, 31 (2021).

486 28. Edelstein, A., Amodaj, N., Hoover, K., Vale, R. \& Stuurman, N. Computer control of 487 microscopes using $\mu$ Manager. Curr. Protoc. Mol. Biol. Chapter 14, Unit14.20 (2010).

488 29. Stuart, T. et al. Comprehensive Integration of Single-Cell Data. Cell 177, 1888-1902.e21 489 (2019).

490 30. Wolf, F. A., Angerer, P. \& Theis, F. J. SCANPY: large-scale single-cell gene expression 491 data analysis. Genome Biol. 19, 15 (2018). 


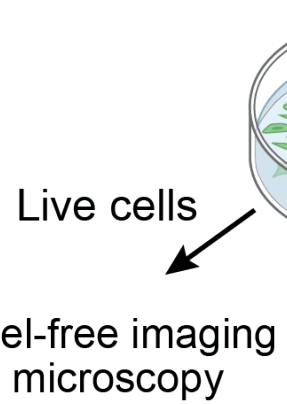

(1) Live-cell label-free imaging with Raman microscopy

(2) scRNA-seq
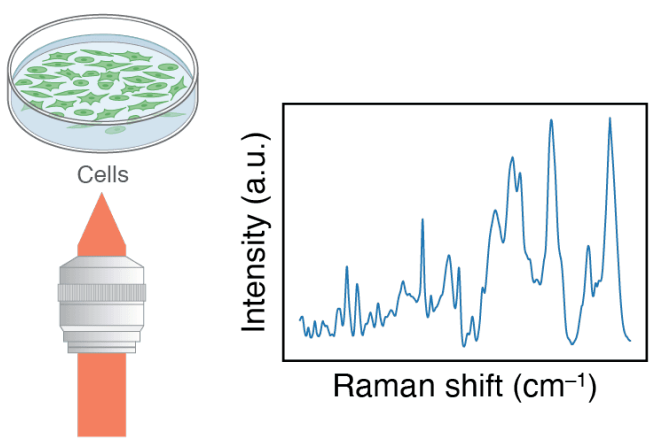

Raman shift $\left(\mathrm{cm}^{-1}\right)$

Raman microscope

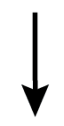

(3) Spatial smFISH for anchoring Raman spectra and scRNA-seq

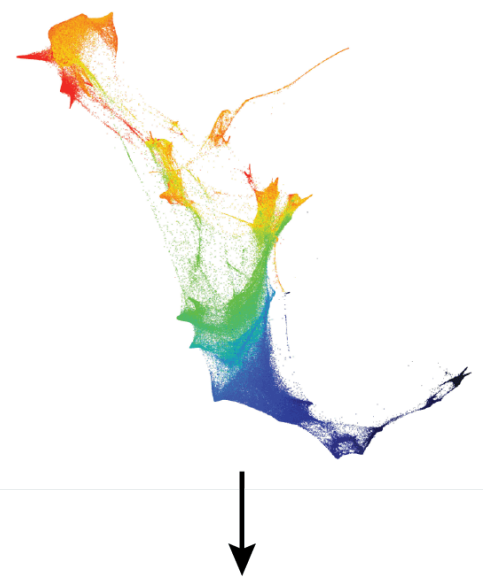

Trajectory analysis and marker gene selection

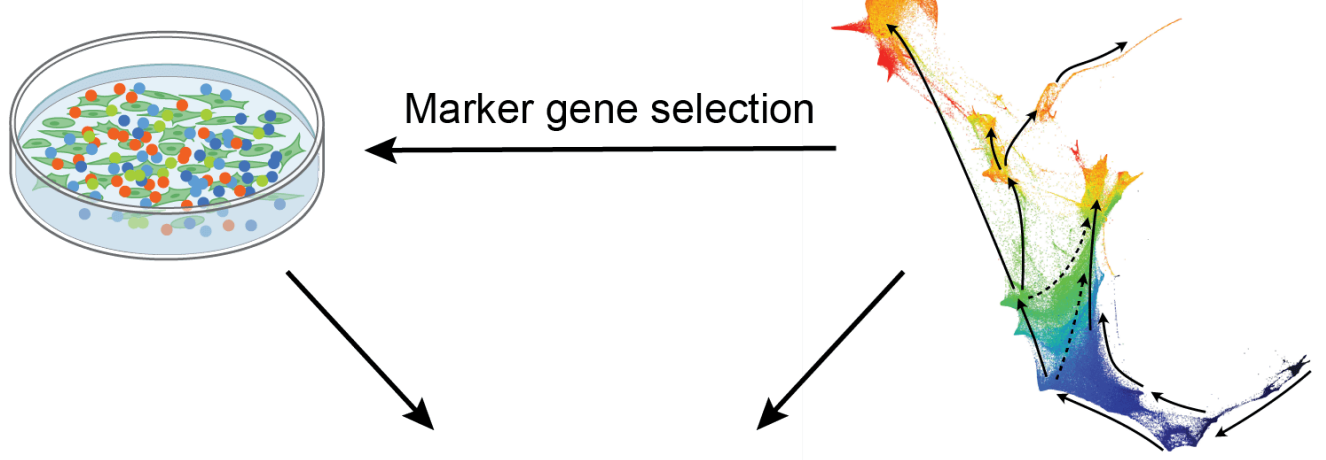

(4) Label-free, non-destructive prediction of single-cell transcriptome profiles via multi-modal data integration and translation

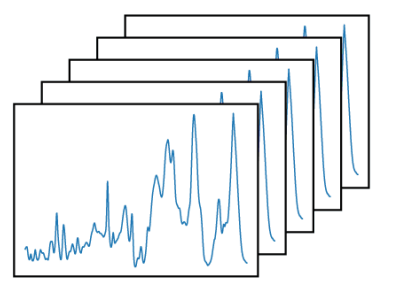

Single cell Raman spectra

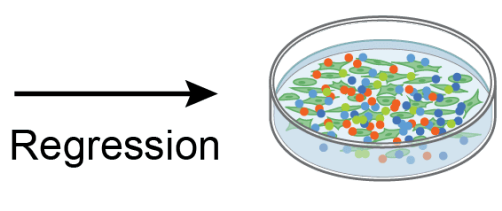

Anchor:smFISH
Integration via

Tangram

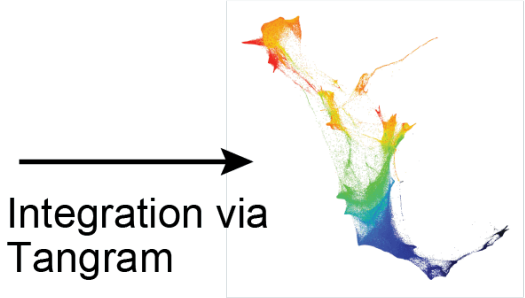

scRNA-seq 
494 Fig. 1 | Raman2RNA. Live cells are cultured on gelatin-coated quartz glass-bottom plates (top) and 495 Raman spectra are then measured at each pixel (at spatial sub-cellular resolution) within an image frame 496 (1), followed by smFISH imaging in the same area (3). From parallel plates, cells are dissociated into a 497 single cell suspension and profiled by scRNA-seq (2). scRNA-seq profiles are used to select 9 marker 498 genes for 5 major cell clusters, and those are measured with spatial smFISH (3). Lastly, a regression 499 model is trained (4) to predict anchor smFISH profiles from Raman spectra, followed by integration via 500 Tangram $^{1}$ to predict whole single-cell transcriptome profiles from smFISH profiles. 
Fig. 2

a

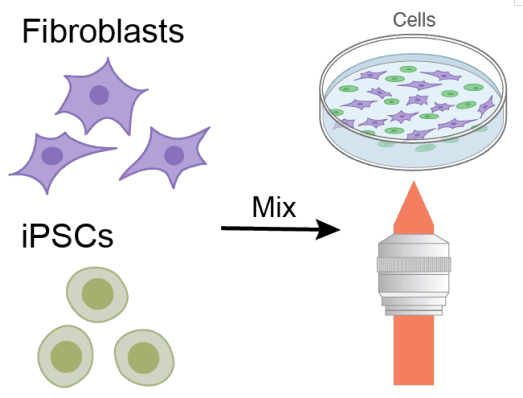

iPSC/fibroblast mixture experiment
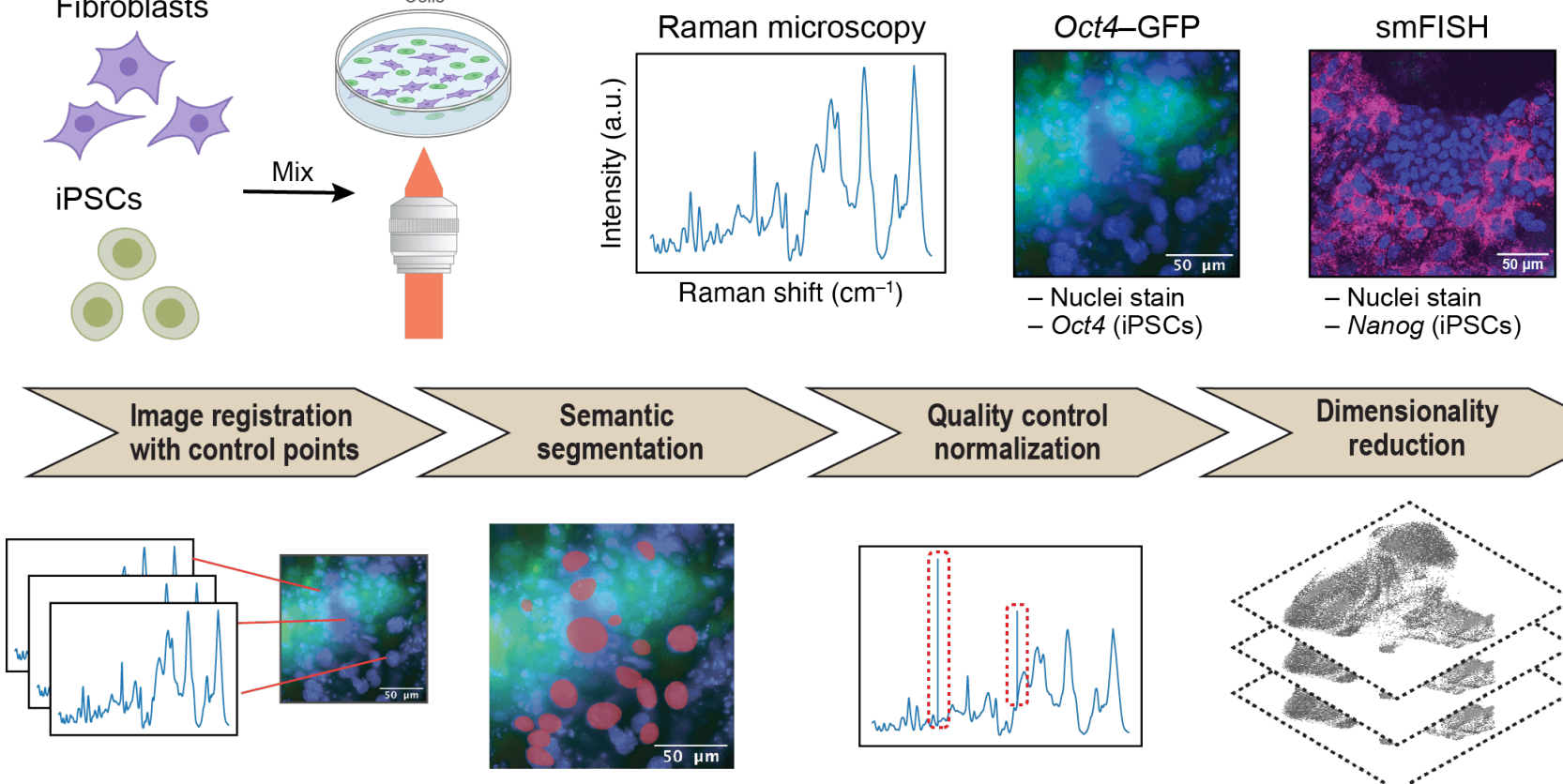

b
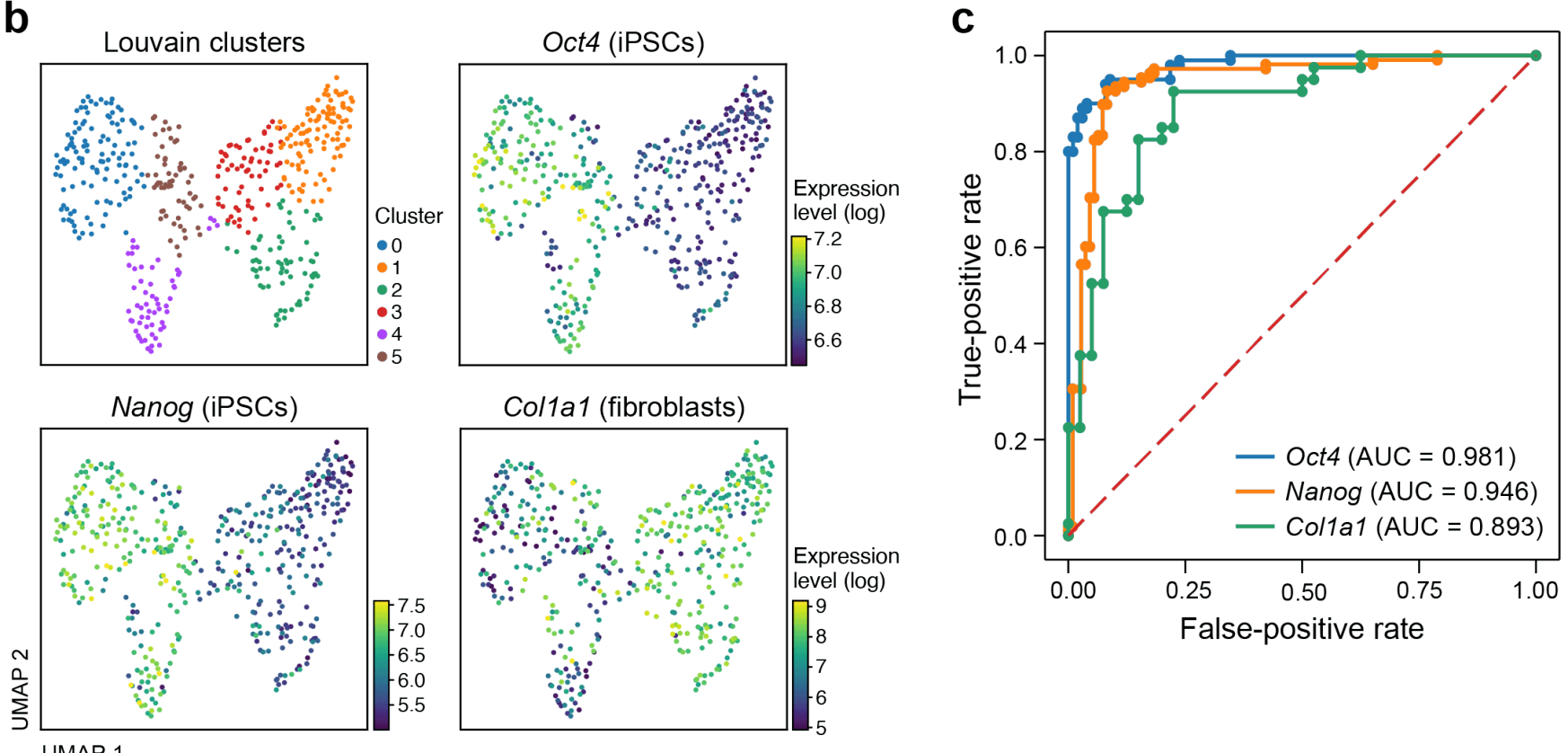

501

Fig. 2 | Raman2RNA accurately distinguishes cell types and predicts binary expression of marker genes in a mixture of mouse fibroblasts and iPSCs. a. Overview. Top: Experimental procedures.

504 Mouse fibroblasts and iPSCs were mixed 1:1 and plated on glass-bottom plates, followed by Raman

505 imaging of live cells, nuclei staining and measurement of endogenous Oct4-GFP (iPSC marker) reporter)

506 by fluorescence imaging, and cell fixation and processing for smFISH with DAPI and probes for Nanog 
507 (iPSCs, magenta) and Collal (fibroblasts). Bottom: Preprocessing and analysis. From left: Image

508 registration with control points (Methods), was followed by semantic cell segmentation, outlier

509 removal/normalization and dimensionality reduction. b. Raman2RNA distinguishes cell states from

510 Raman spectra. 2D UMAP embedding of single-cell Raman spectra (dots) colored by Louvain clustering

511 labels (top left) or smFISH measured expression of Oct4 (top right), Nanog (bottom left) and Colla1

512 (bottom right). c. Raman2RNA accurately predicts binary (on/off) expression of marker genes. Receiver

513 operating characteristic (ROC) plots and area under the curve (AUC) obtained by classifying the 'on' and

514 'off' states of Oct4 (blue), Nanog (orange) and Collal (green). 
a

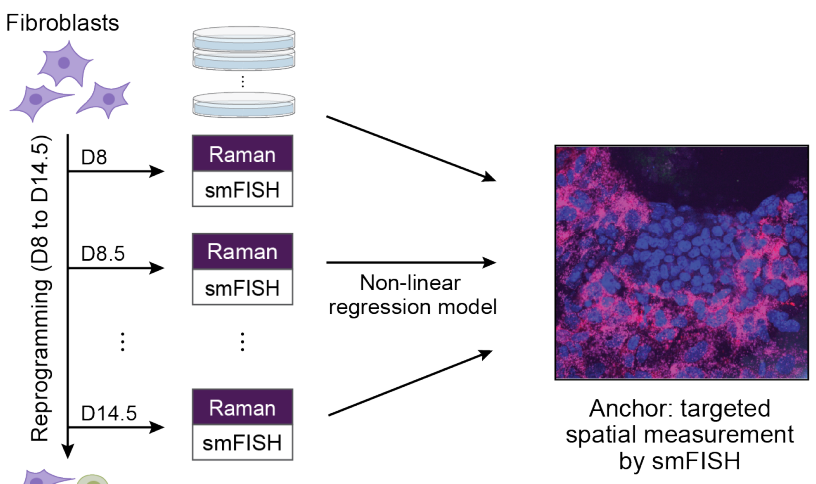

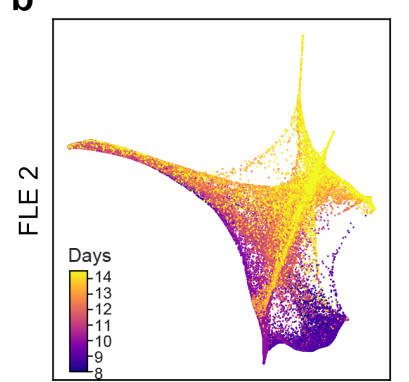

FLE 1

f

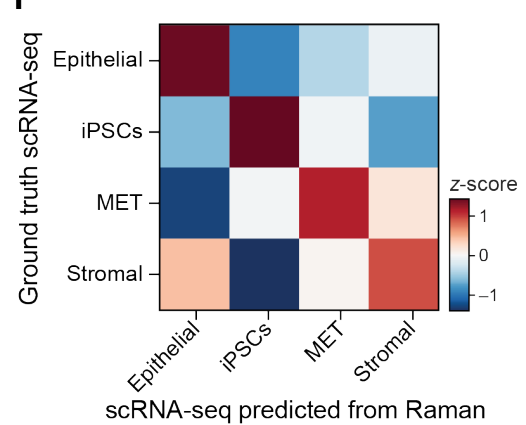

i

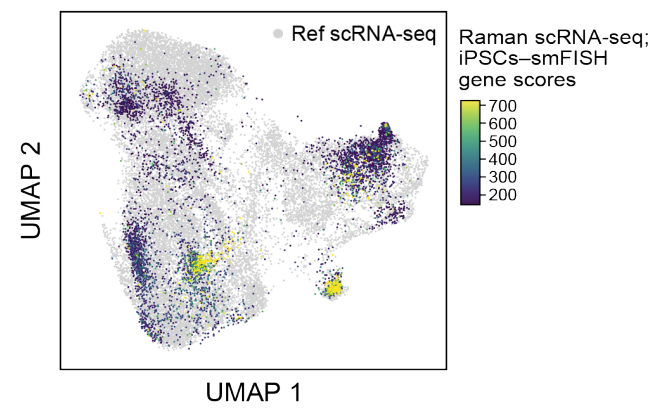

g
C

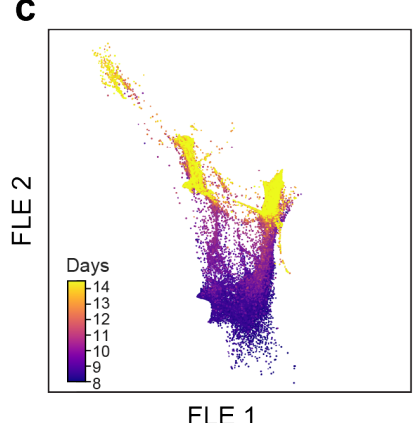

UMAP 1

j

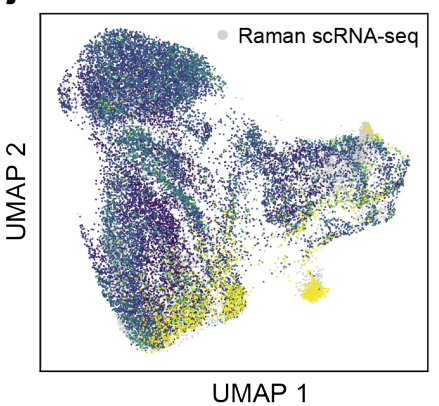

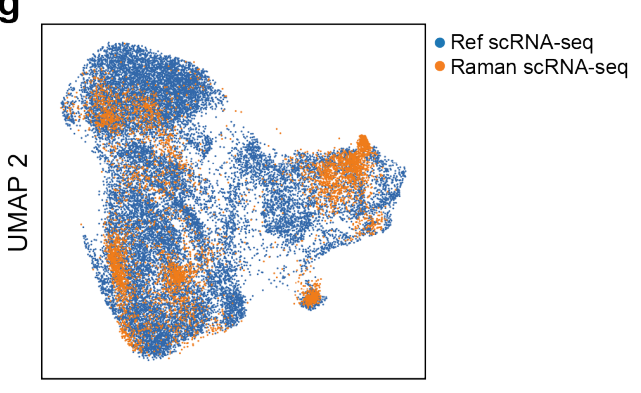

Fig. 3
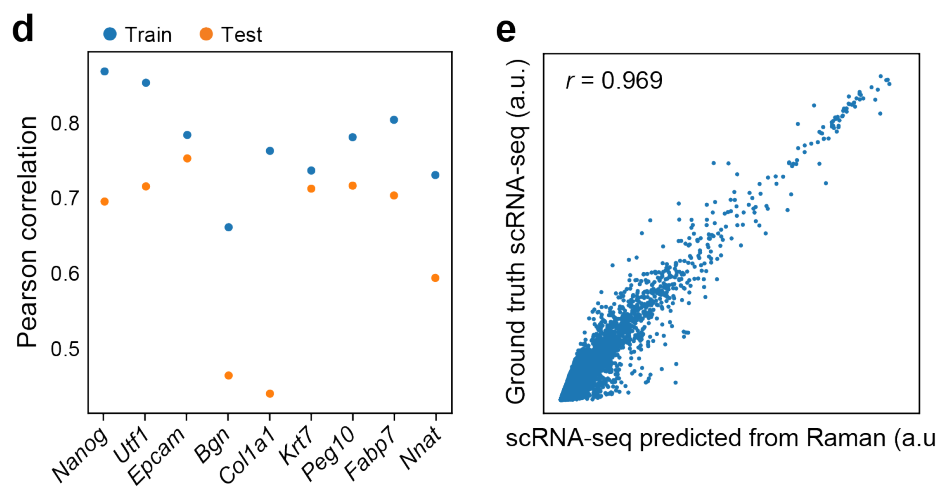

scRNA-seq predicted from Raman (a.u.)

h

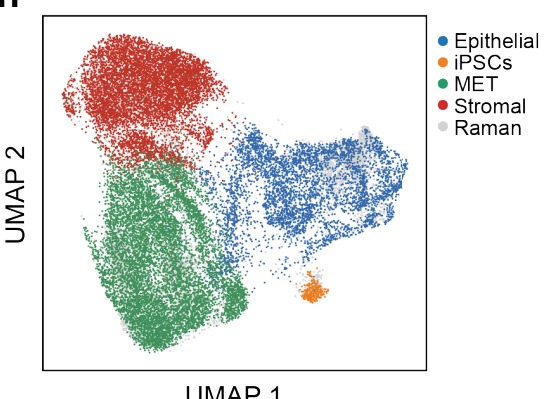

UMAP 1

k

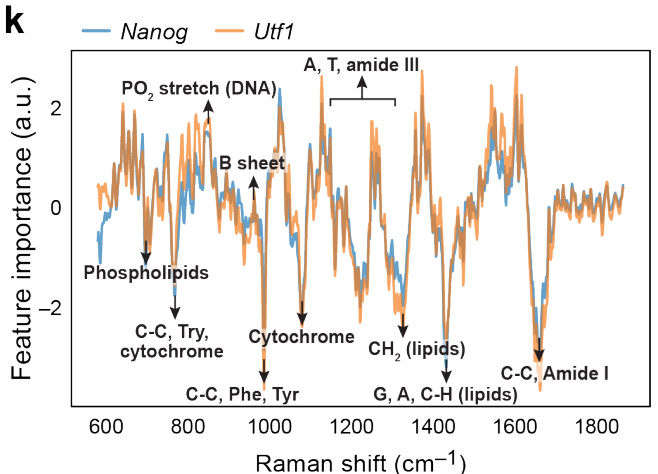

516 Fig. 3 | Raman2RNA predicts single-cell RNA profiles across cell types during reprogramming of

517 mouse fibroblasts to iPSCs. a. Approach overview. From left: Mouse fibroblasts were reprogrammed 
518 into induced pluripotent stem cells (iPSCs) over the course of 14.5 days (' $D$ '), and, at half-day intervals

519 from days 8 to 14.5, spatial Raman spectra, smFISH for nine anchor genes, and nuclei stain by

520 fluorescence imaging were measured for each plate. Machine learning and multi-modal data integration

521 methods (Catboost and Tangram) were used to predict single-cell RNA-seq profiles from Raman spectra

522 using smFISH as anchor. b,c. Low dimensionality embedding of single-cell Raman spectra captures

523 progress in reprogramming. Force-directed layout embedding (FLE) of Raman spectra (b, dots) or

524 scRNA-seq (c, dots) colored by days of measurement (colorbar). d. Correct prediction of smFISH anchors

525 from Raman spectra. Pearson correlation coefficient ( $y$ axis) between measured (smFISH) and Raman-

526 predicted levels for each smFISH anchor ( $x$ axis) in leave-one-out cross-validation where 8 out of 9

527 smFISH anchor genes were used for training, and the left-out gene was predicted. e.f. Raman2RNA

528 accurately predicts pseudo-bulk expression profiles of major cell types. e. scRNA-seq measured (y axis)

529 and R2R-predicted (x axis) for each gene (dot) in pseudo-bulk RNA profiles averaged across iPSCs. f.

530 Pair-wise correlation (color bar) between Raman-predicted and scRNA-seq measured pseudo-bulk

531 profiles in each cell types (rows, columns). g-j. Co-embedding highlights agreement between real and

532 R2R inferred single cell profiles. UMAP co-embedding of Raman predicted RNA profiles and measured

533 scRNA-seq profiles (dots) colored by data source (g, Raman predicted in orange; measured scRNA-seq in

534 blue), cell type annotations (h) or by iPSC gene signature scores (calculated by averaging expression of

535 genes Nanog and Utfl, and subtracting the average of a randomly selected set of reference genes;

536 Methods) of Raman-predicted profiles (i) or of real scRNA-seq (j). k. Feature importance scores of

537 Raman spectra in predicting expression profiles. Feature scores for iPSC related marker genes (y axis)

538 along the Raman spectrum (x axis). Known Raman peaks ${ }^{2}$ were annotated. 


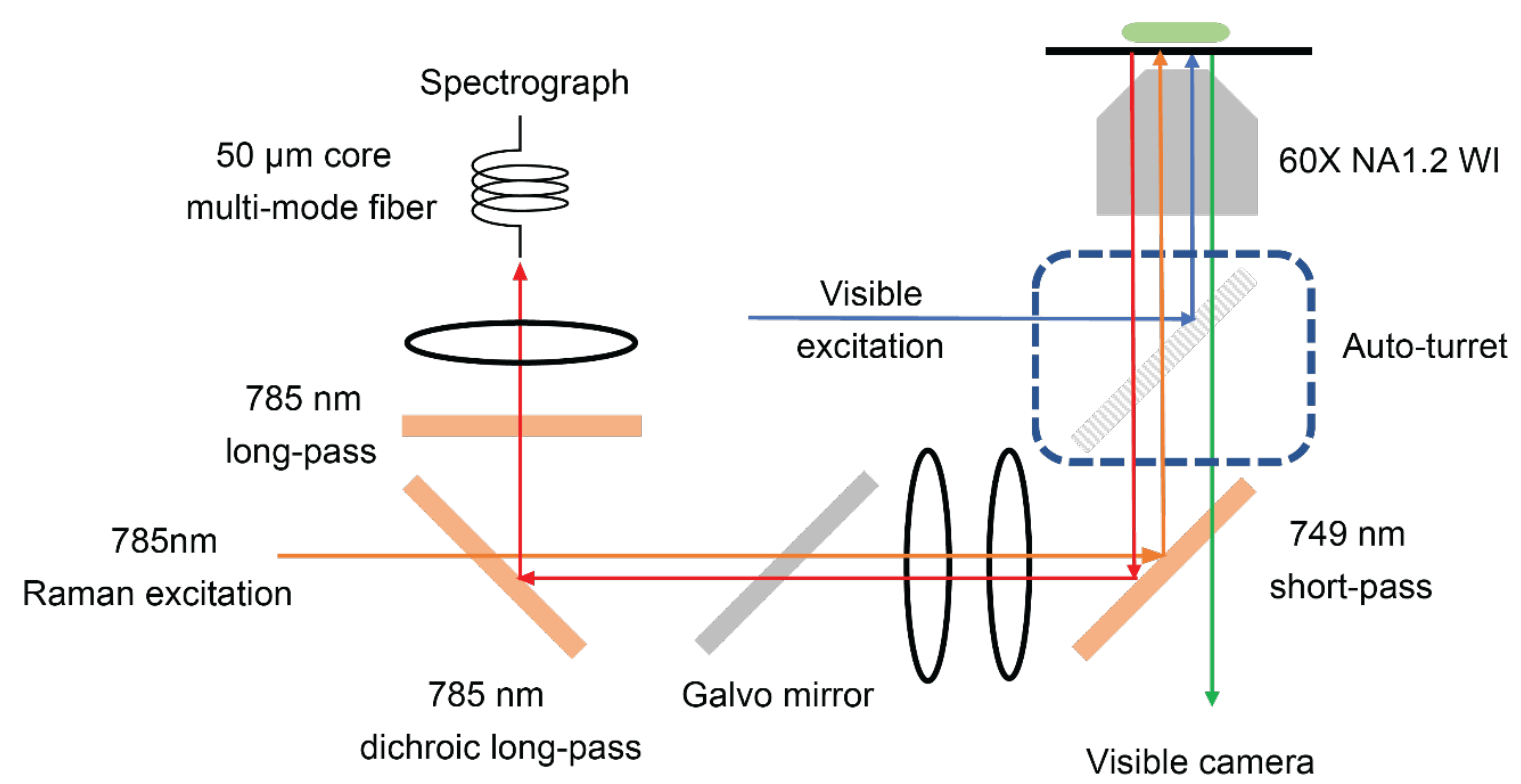

558 Extended Data Fig. 1 | A multi-modal Raman microscope capable of fluorescence imaging and

559 Raman microscopy. Schematic of a Raman microscope integrated with a wide-field fluorescence

560 microscope for simultaneous detection of nuclei staining, bright field, fluorescence channels, and Raman 561 images. 
Microscope control software

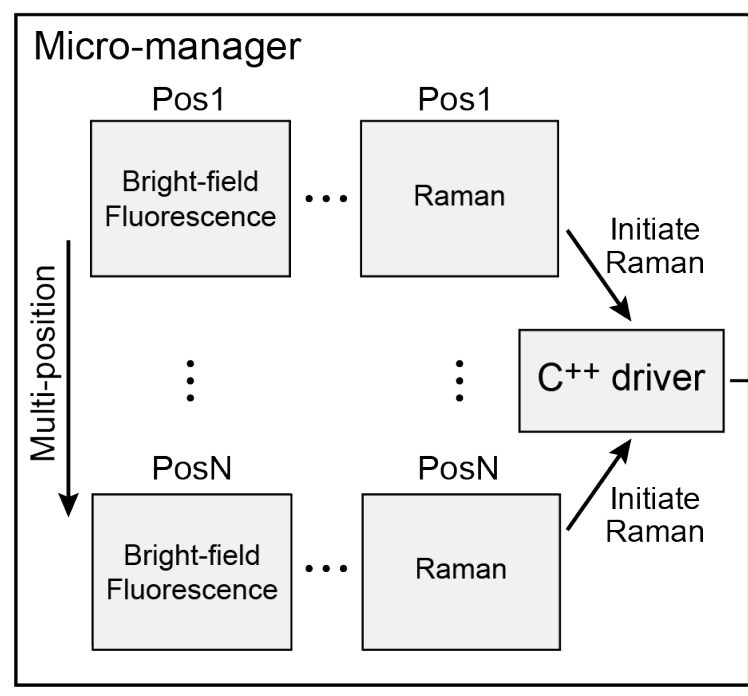

Raman scanning

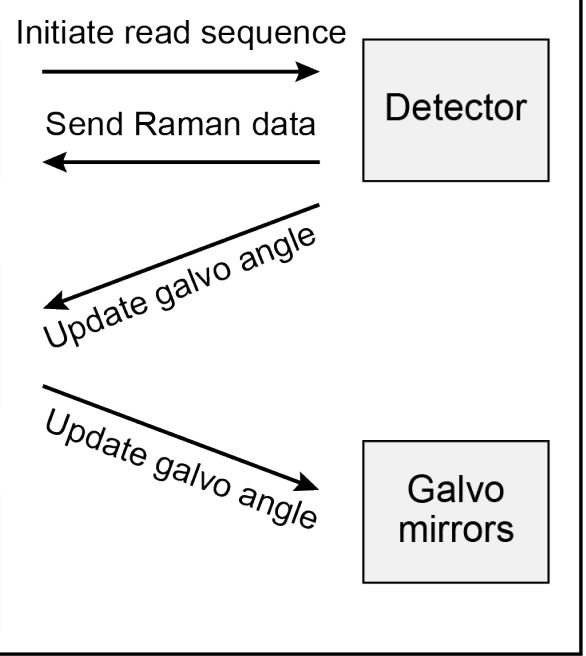

Extended Data Fig. 2 | Overview of high-throughput Raman imaging software used in the study. A registered as a 'dummy' channel along with brightfield and fluorescence channels. Micro-manager was responsible for changing the field of view (FOV) and imaging modality. During the Raman sequence,

572 Micro-manager communicated with a digital acquisition (DAQ) board, through which a transistor-to-

573 transistor logic (TTL) signal was generated to initiate the scanning sequence. Upon detection of the TTL

574 signal, the MATLAB script controlled the Raman detector, laser shutter, and updated the galvo mirror

575 angles through the DAQ board. 


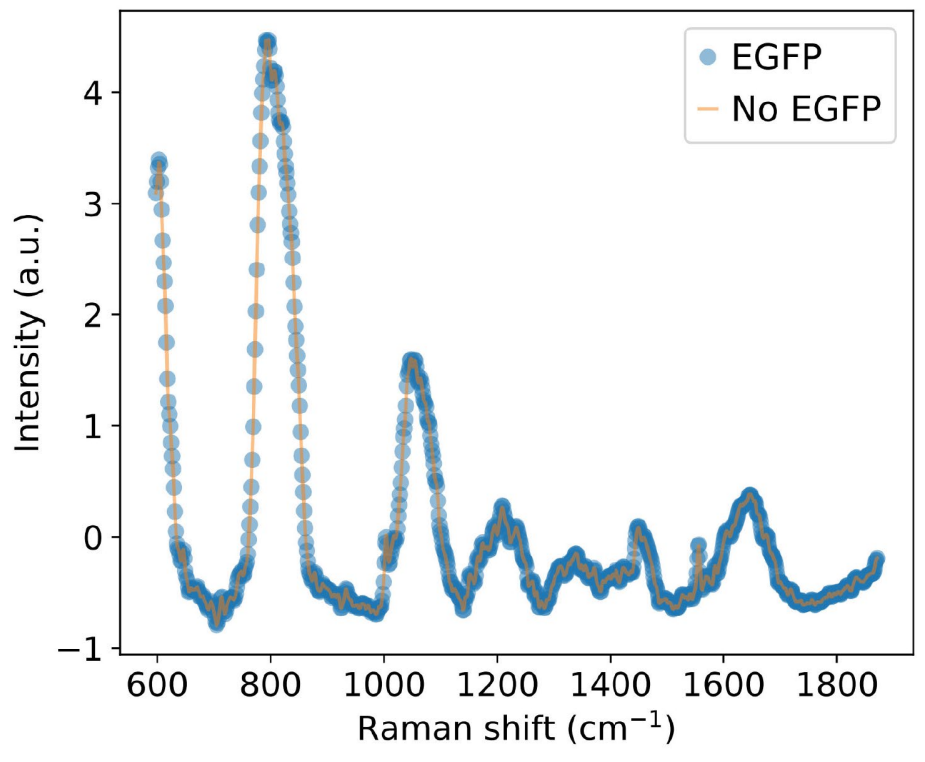

596

597 Extended Data Fig. 3 | GFP does not interfere in Raman spectra measurement. Raman spectra of 598 culture media with (blue) and without (orange) GFP at physiological concentration. 


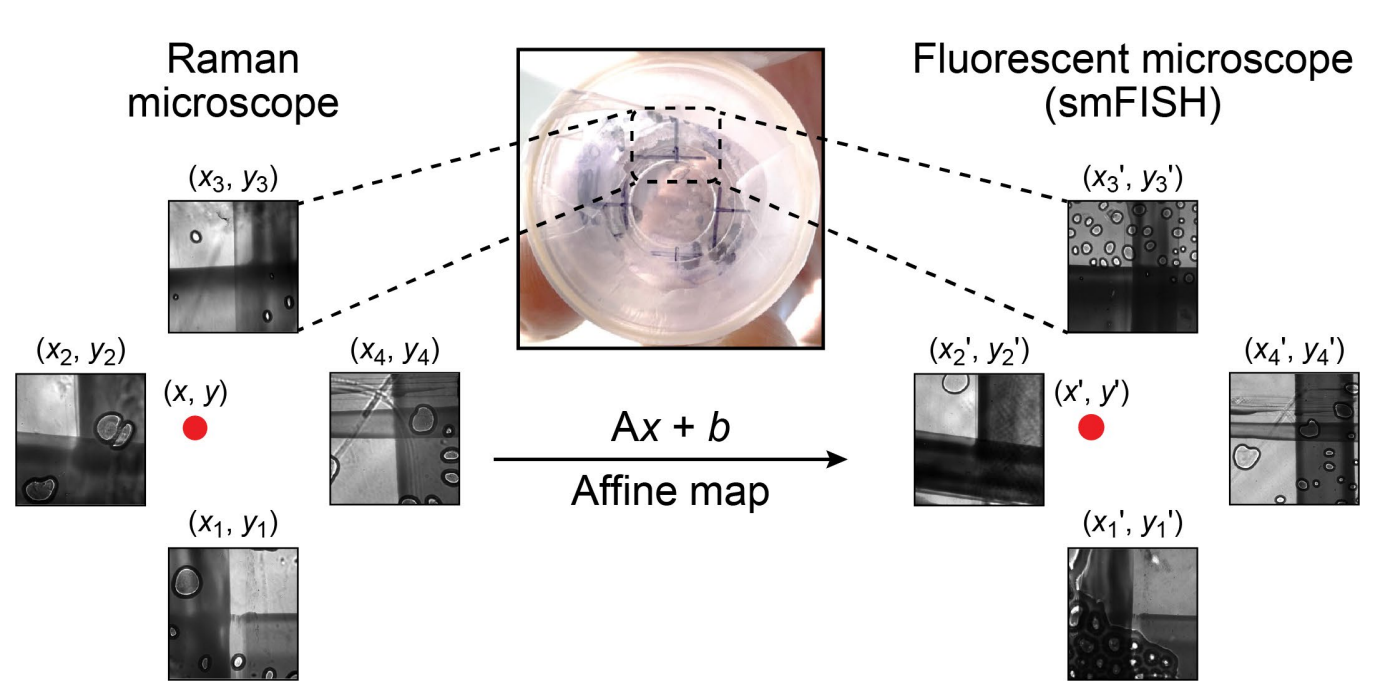

618 control points. Control points were inscribed under petri dishes with permanent markers and the

619 coordinates were measured prior to any data acquisition. After Raman measurement and smFISH

620 processing, samples were placed back to the microscope and control point coordinates were remeasured.

621 Then, affine mapping was used to update the FOV coordinates to locate the exact same cells. 

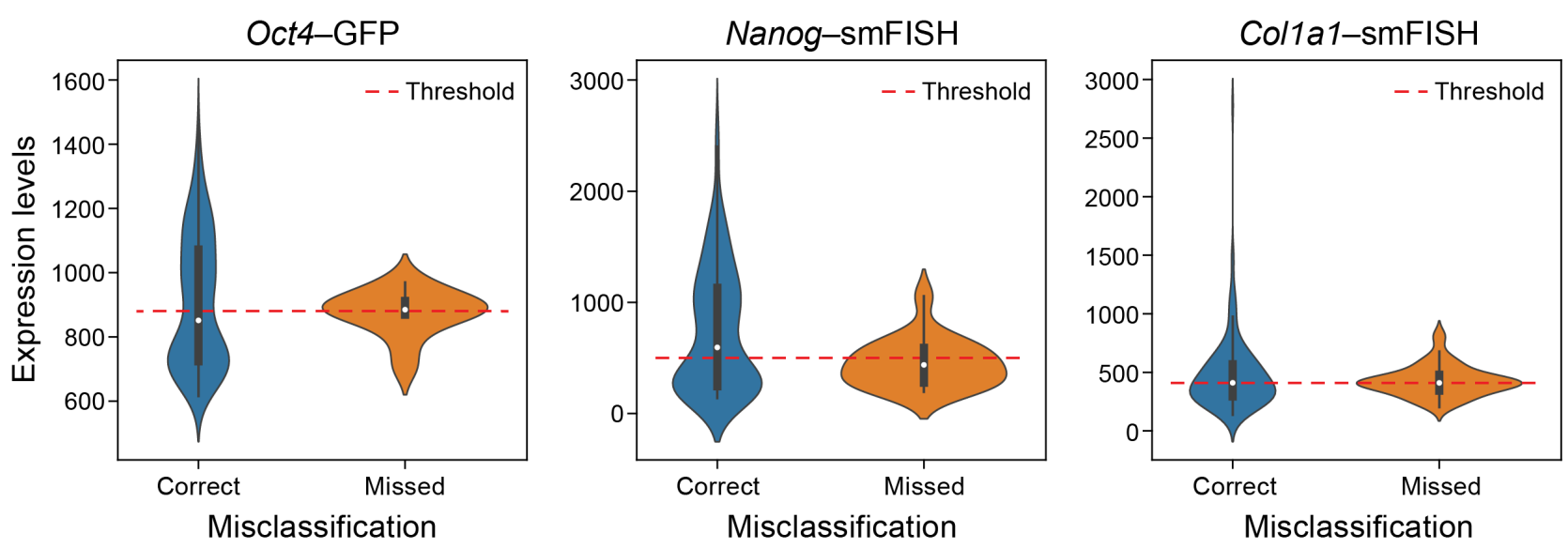
classifier. 

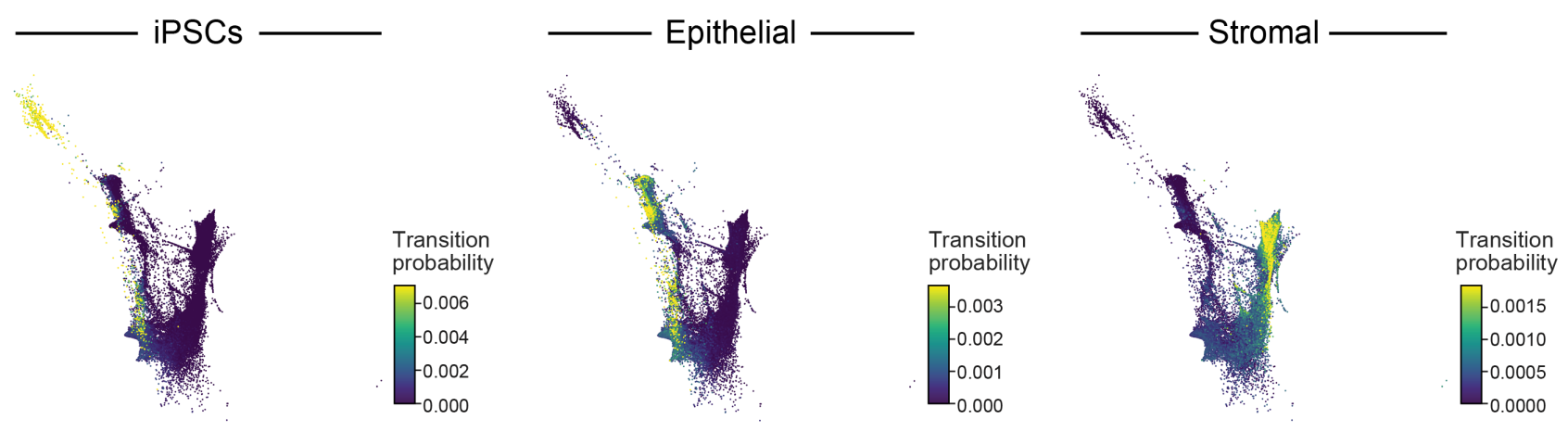

643 Extended Data Fig. 6 | Cell transition probabilities inferred by Waddington-OT from scRNA-seq

644 during reprogramming. Force-directed layout embedding (FLE) of scRNA-seq profiles (dots) from

645 days 8 to 14.5 of reprogramming (dots) colored by the transition probability of each cell as inferred by

646 Waddington-OT to be an ancestor of iPSCs (left), epithelial cells (middle) or stromal cells (right) at day 64714.5 .

648 

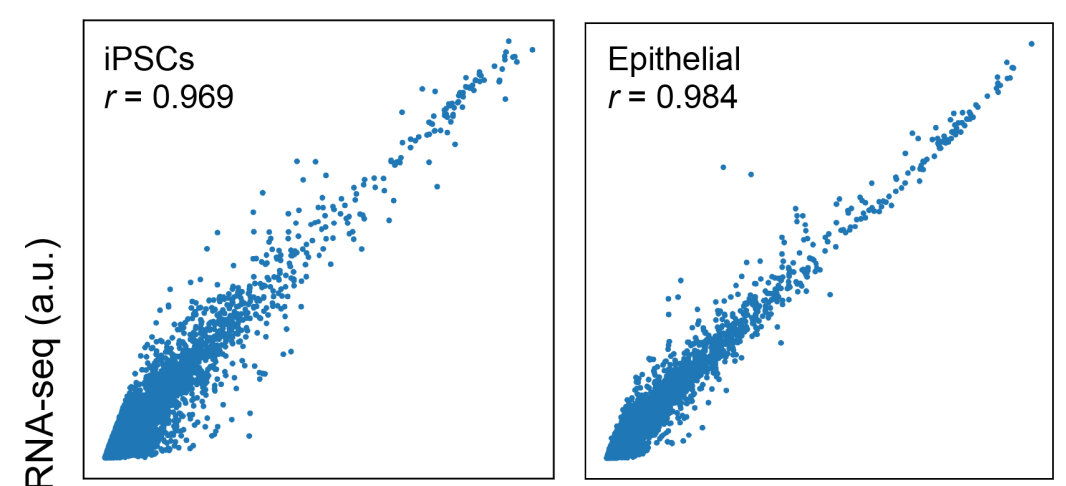

669

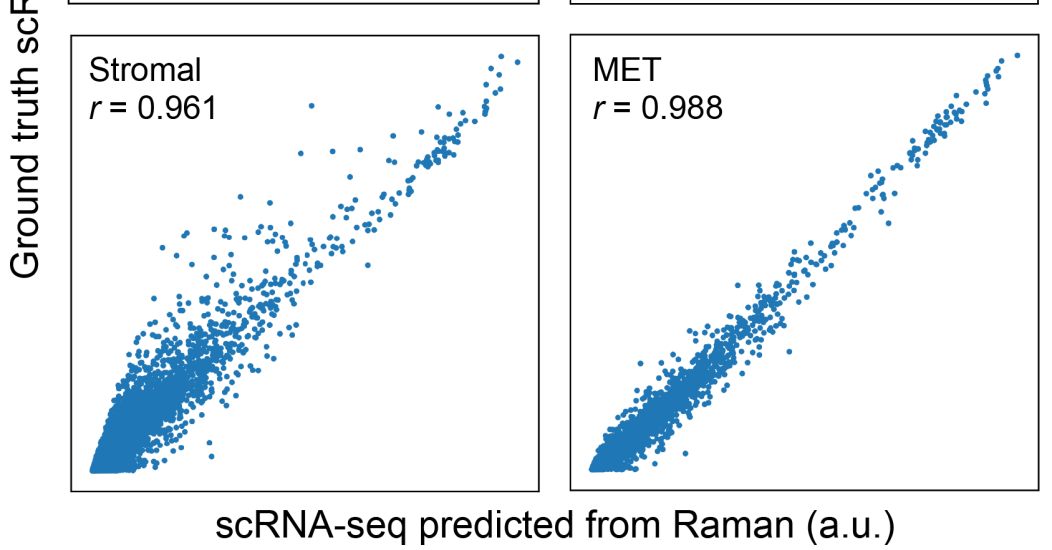

670

671 Extended Data Fig. 7 | Raman-predicted and scRNA-seq measured pseudo-bulk profiles are well

672 correlated across cell types. ScRNA-seq measured (y axis) and R2R-predicted ( $\mathrm{x}$ axis) expression for

673 each gene (dot) in pseudo-bulk RNA profiles averaged across cells labeled as iPSC (top left), epithelial

674 (top right), stromal (bottom left) and MET (bottom right). Pearson's $r$ is denoted at the top left corner. 

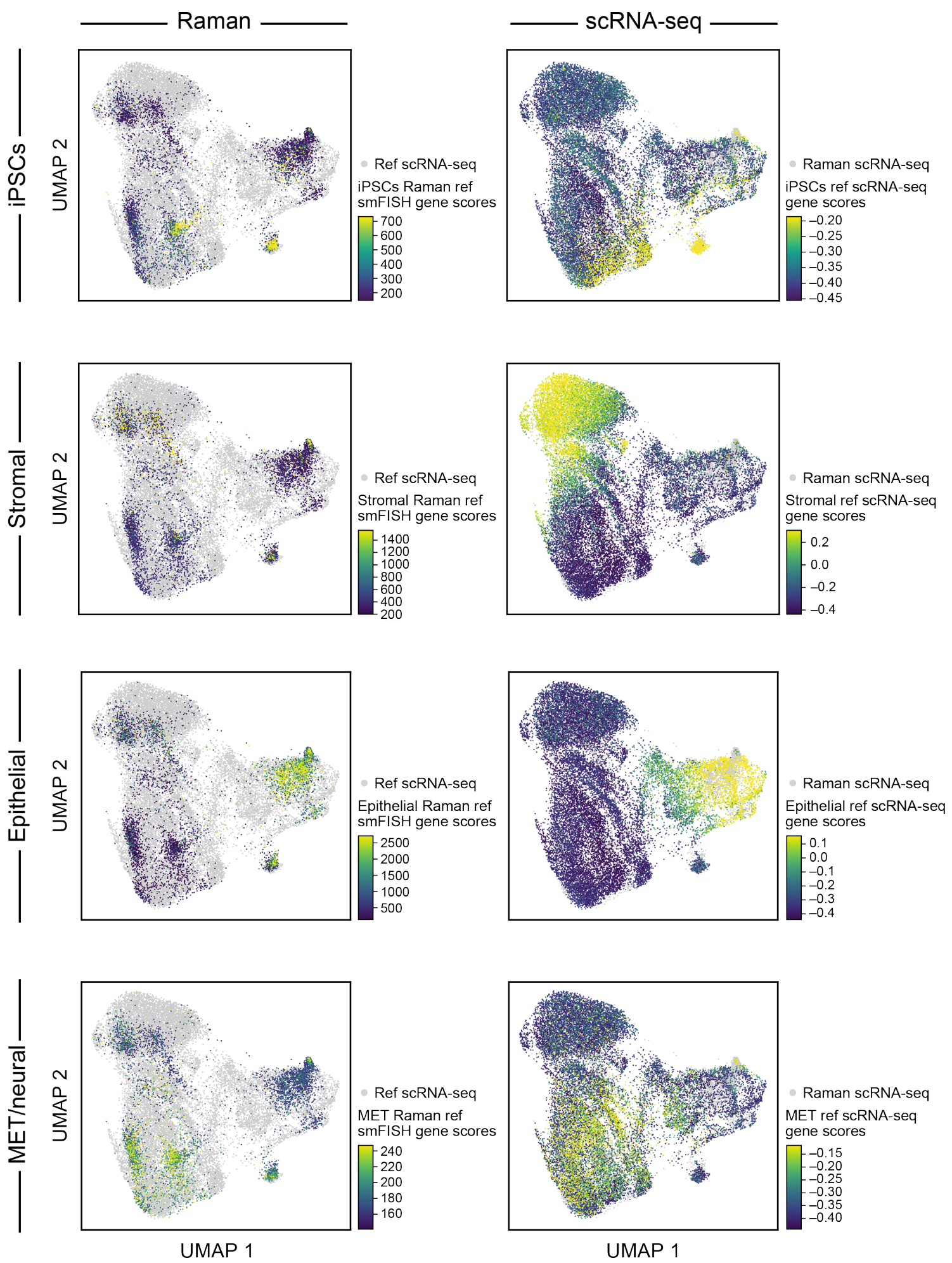

Extended Data Fig. 8 | Measured and Raman-predicted single cell profiles co-embed well as measured scRNA-seq profiles (dots) colored by scores of marker gene for different cell types (rows) 
bioRxiv preprint doi: https://doi.org/10.1101/2021.11.30.470655; this version posted December 1, 2021. The copyright holder for this preprint

(which was not certified by peer review) is the author/funder, who has granted bioRxiv a license to display the preprint in perpetuity. It is made available under aCC-BY-NC-ND 4.0 International license.

682 determined by smFISH measurements (left, for cells with Raman-predicted profiles) or real scRNA-seq

683 measurements (right, for cells with scRNA-seq profiles). 

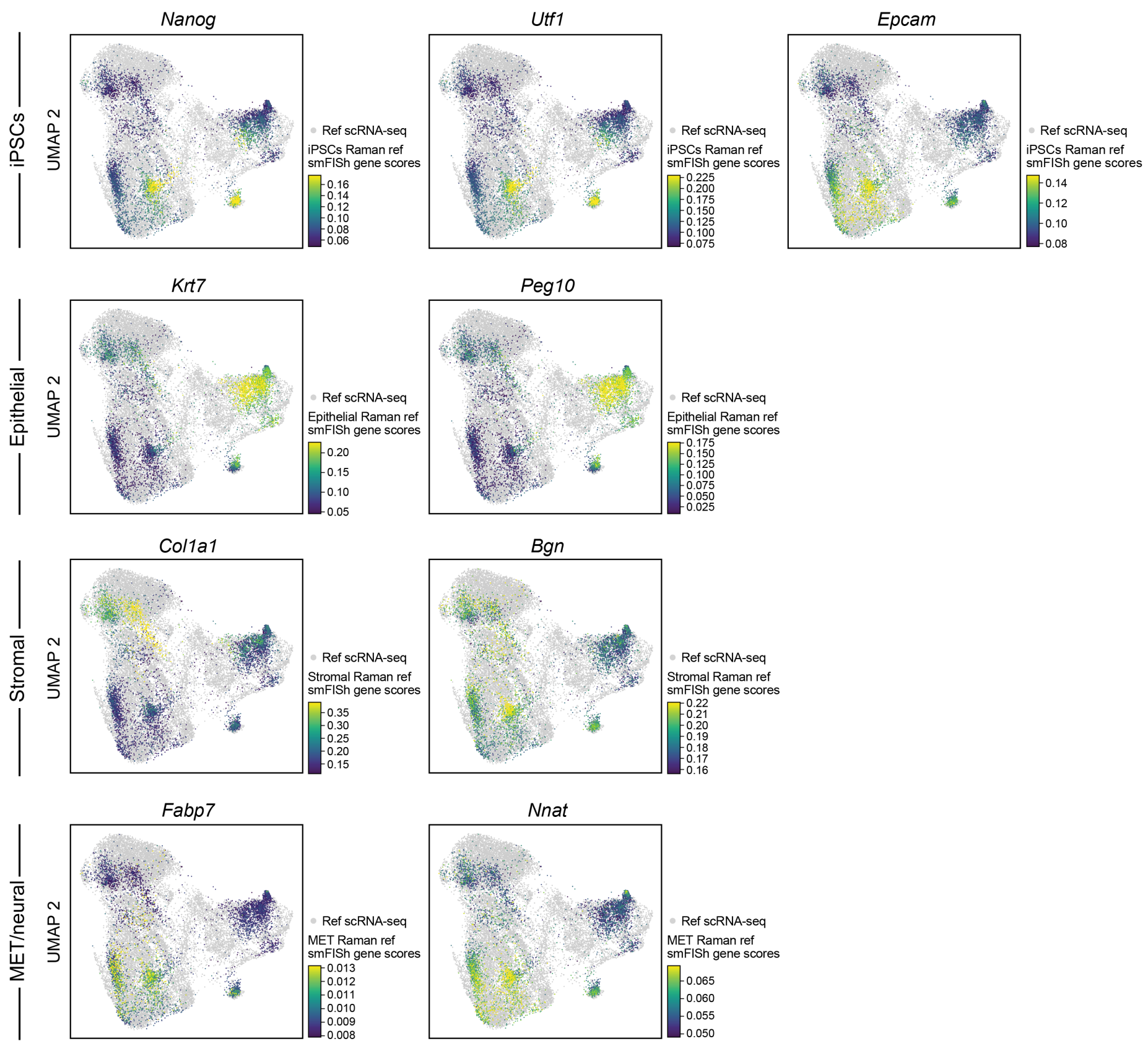

UMAP 1

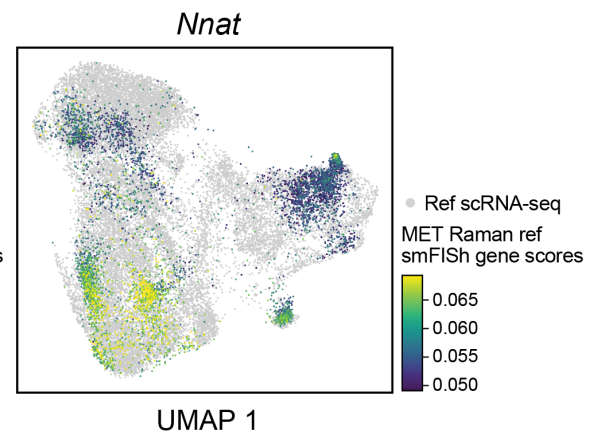

687 Extended Data Fig. 9 | Measured and Raman-predicted single cell profiles co-embed well as

688 reflected by smFISH measurement of Raman cells. UMAP co-embedding of Raman predicted RNA

689 profiles and measured scRNA-seq profiles (dots) where the Raman cells are colored by smFISH

690 measurement of each of nine anchor genes. 

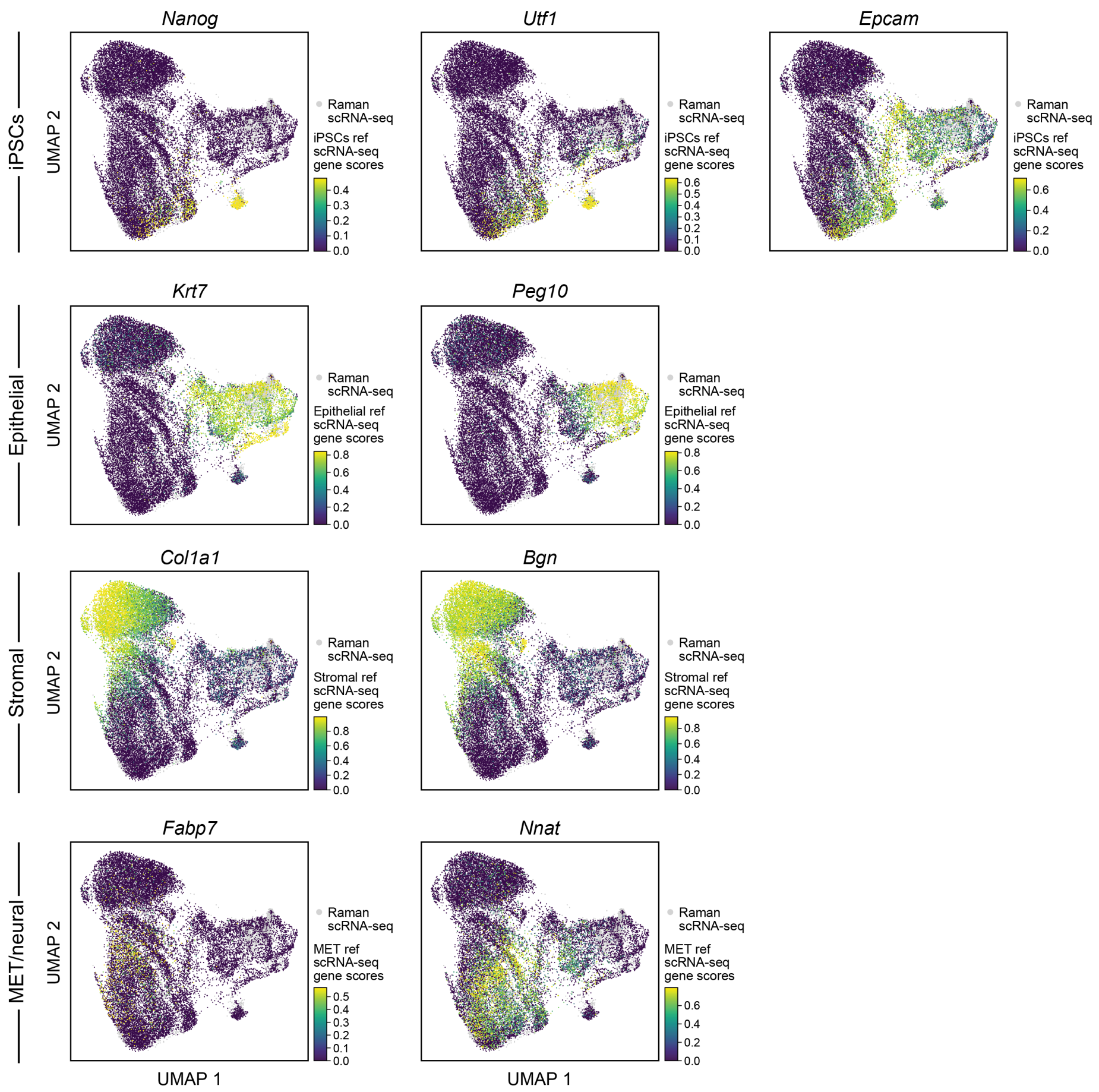

693 Extended Data Fig. 10 | Measured and Raman-predicted single cell profiles co-embed well as

694 reflected by scRNA-seq based expression of nine anchor genes. UMAP co-embedding of Raman

695 predicted RNA profiles and measured scRNA-Seq profiles (dots) where the scRNA-seq profiled cells are

696 colored by scRNA-seq measured expression of each of nine anchor genes. 
Supp. Fig. 11
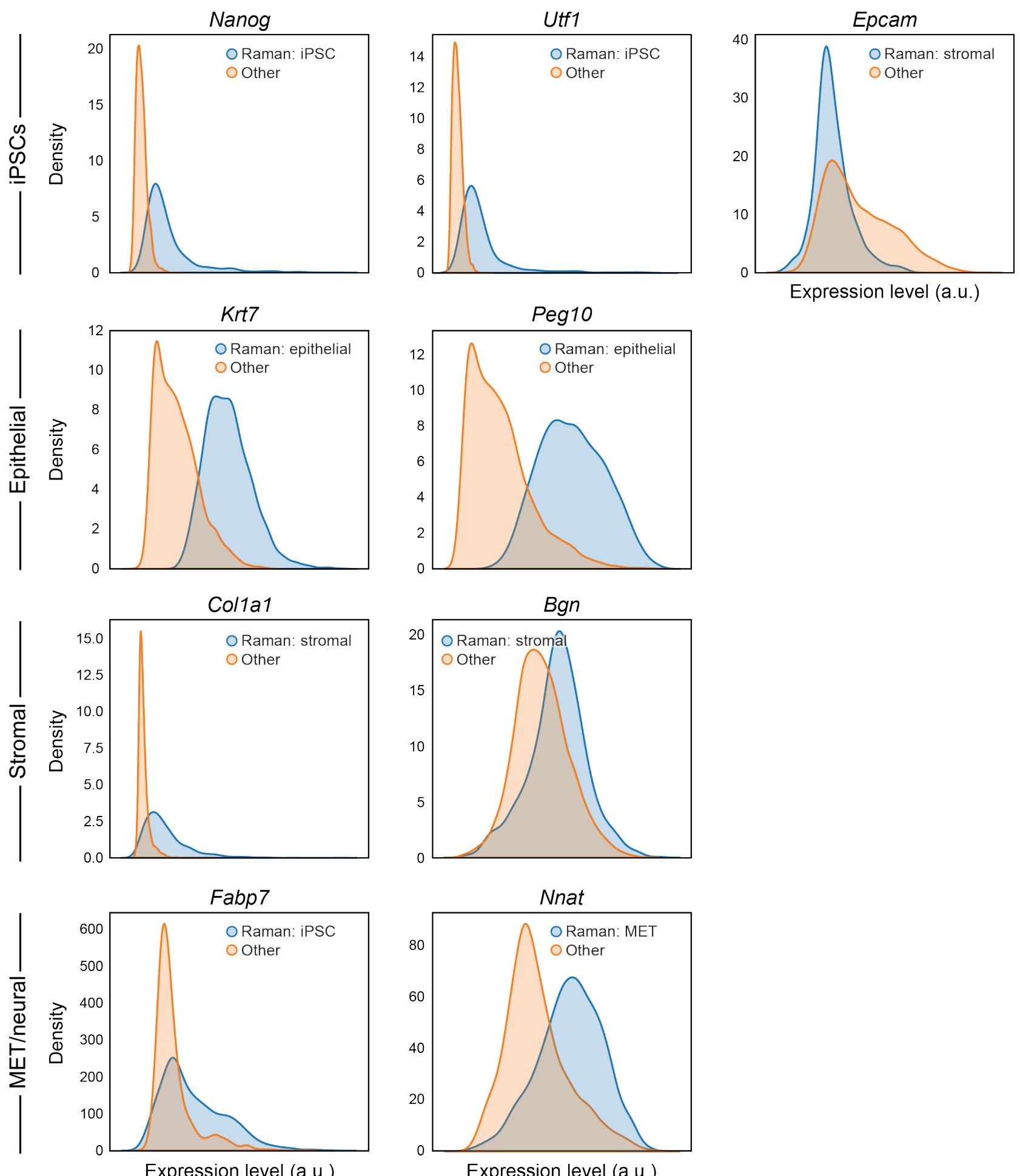

Extended Data Fig. 11 | Distributions of expression of marker genes based on R2R-predicted

profiles. Distributions (density plots) of the predicted expression in Raman2RNA inferred profiles for each marker gene (panel) in its expected corresponding cell type (blue, based on the predicted expression profiles) and all other cells (orange). 

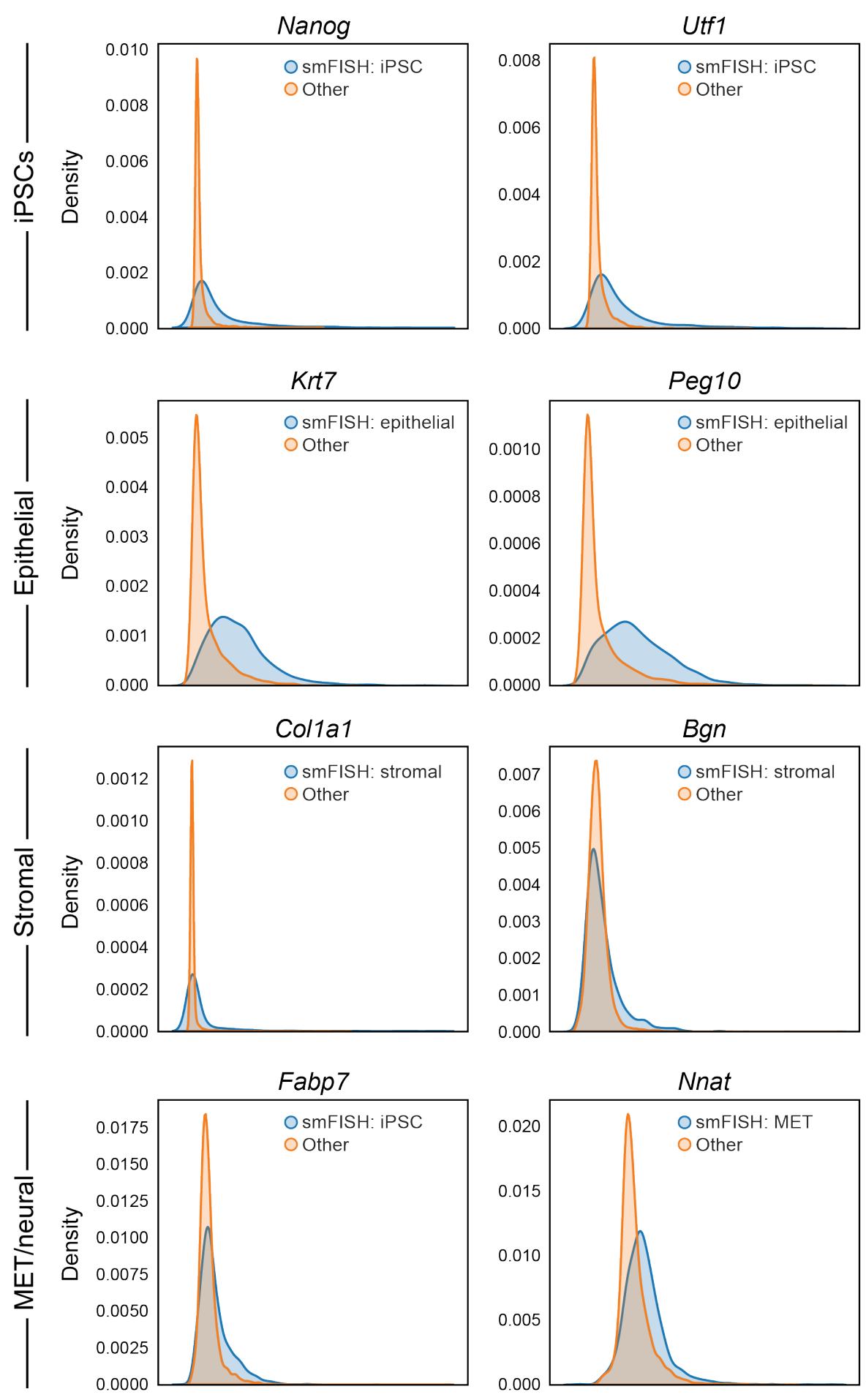
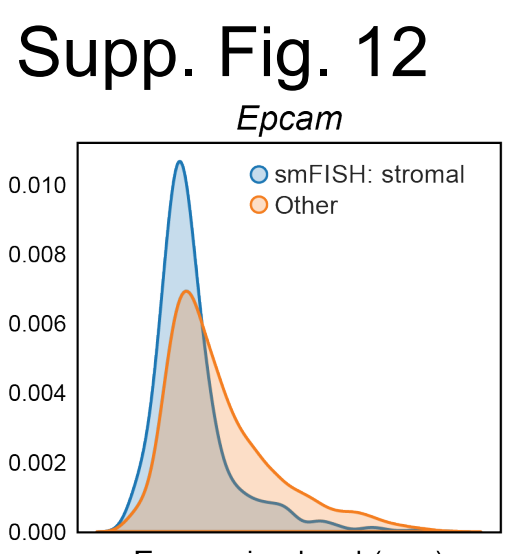

Expression level (a.u.)

Distributions (density plots) of the real smFISH profiles for each marker gene (panel) in its expected

705 (orange). 
- Ref scRNA-seq

- Raman scRNA-seq

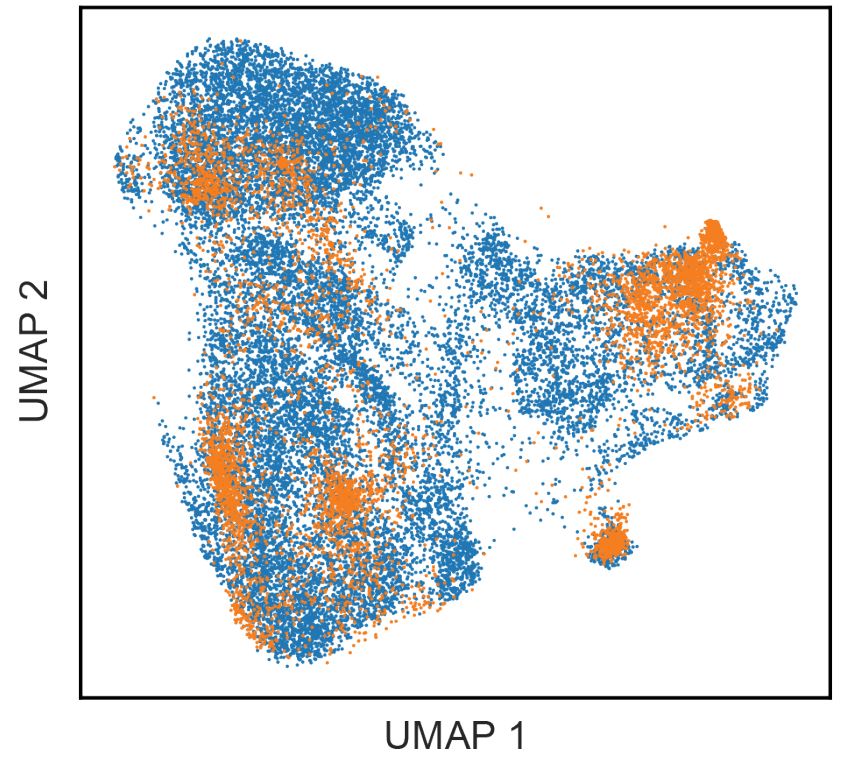

Ref scRNA-seq

smFISH scRNA-seq

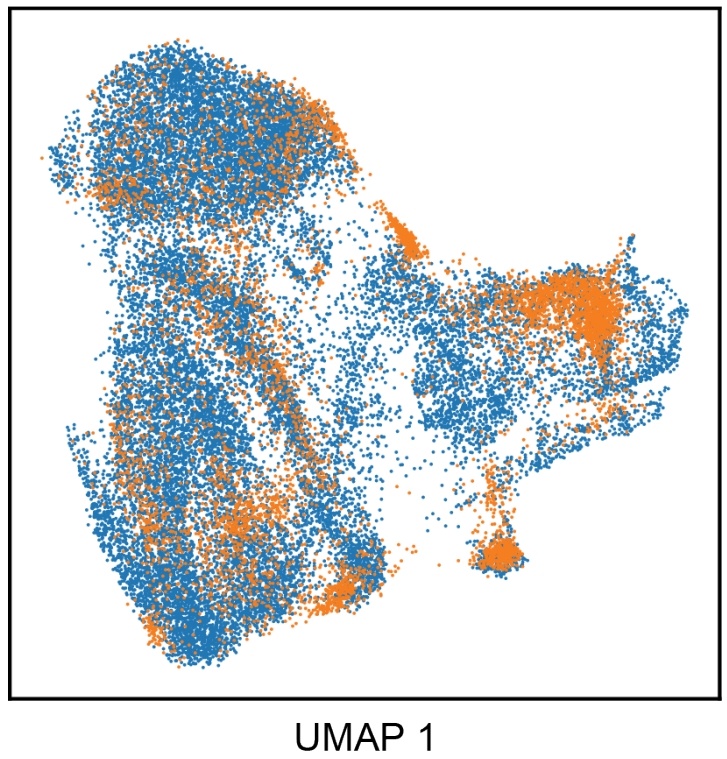

711 and Raman (orange) experiments, with the latter based on either the Raman-predicted RNA profiles (left)

712 or only smFISH-predicted RNA profiles (right). 

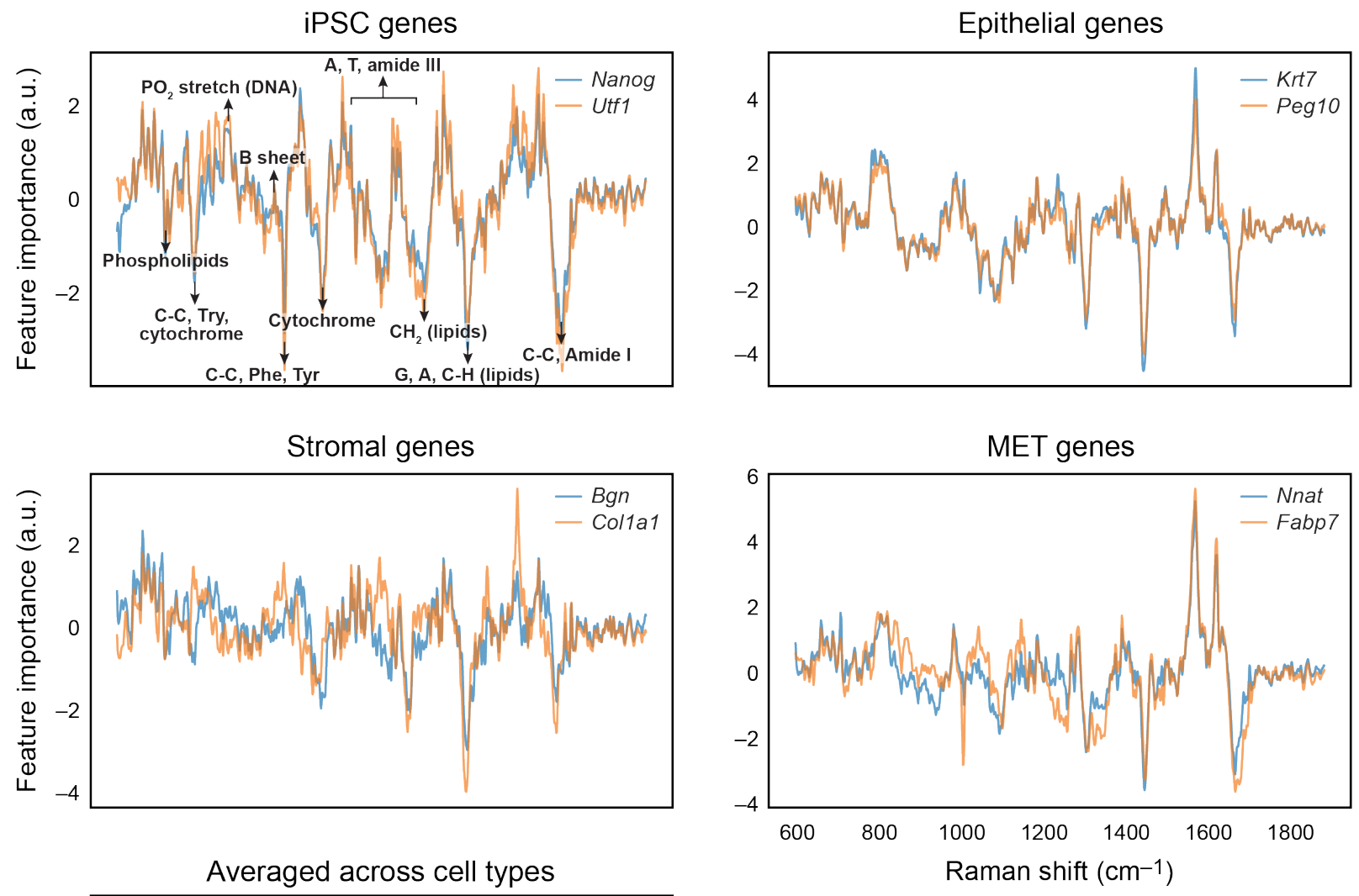

716

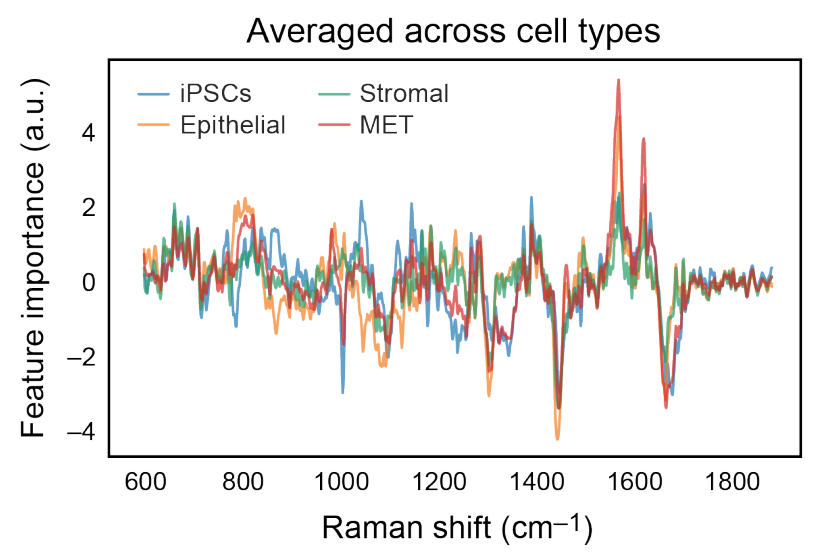

717 Extended Data Fig. 14 | Raman spectral feature importance scores for each smFISH anchor gene

718 and its average across all genes for a cell type. Feature importance scores (y axis) for marker genes of

719 each cell type (top two rows), and for all cell types (bottom row), along the Raman spectrum (x axis).

720 Known signals ${ }^{2}$ are annotated in the top left panel (identical to Fig. 3k). 


\section{Supp. Fig. 15}

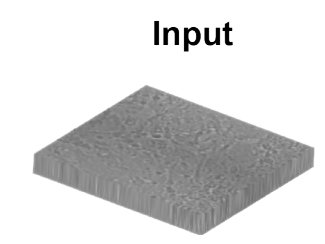

Bright field $z$-stacks

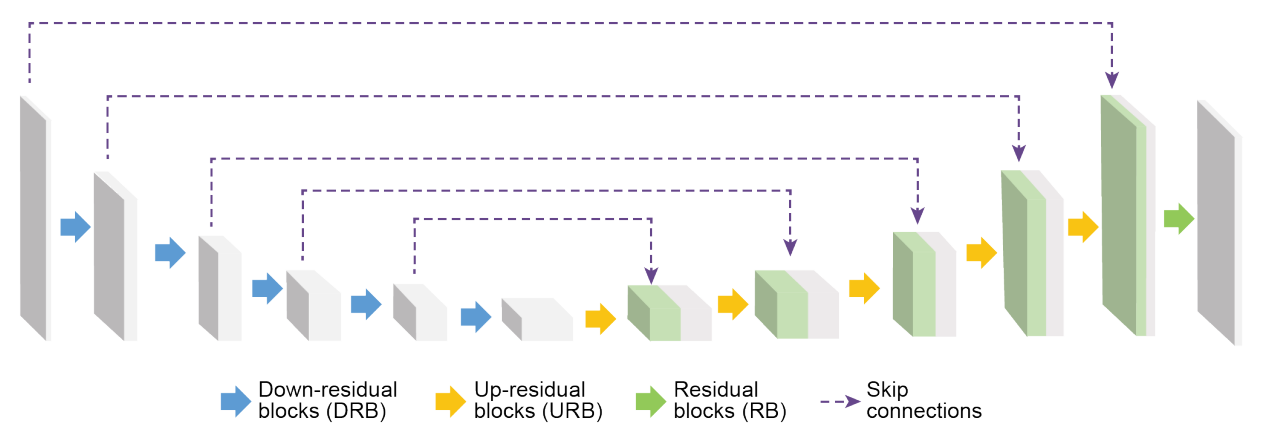

\section{Output}

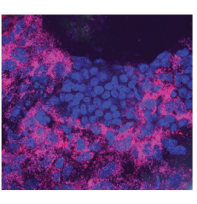

smFISH

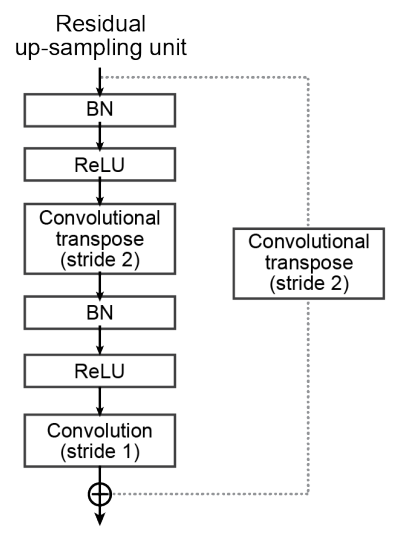

Down-residual

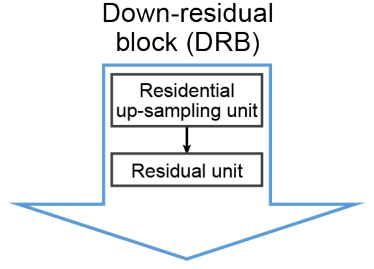

Residual

up-sampling unit

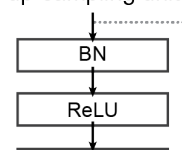

Convolutional Convolution
(stride 2)

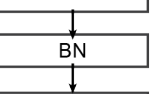

ReLU

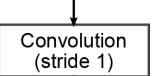

(stride 1)

$\Phi$

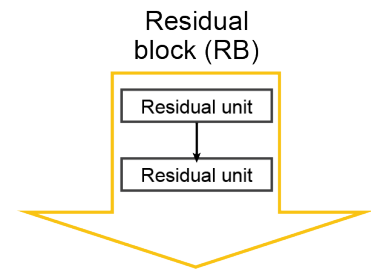

Residual

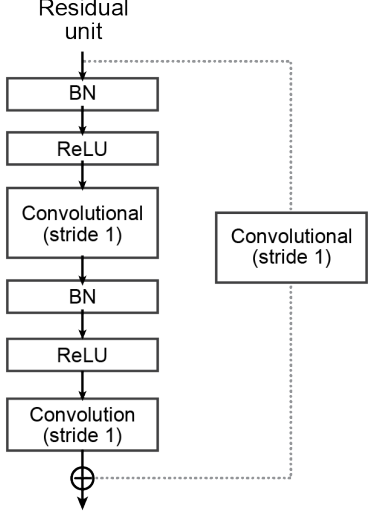

Residual block (URB)

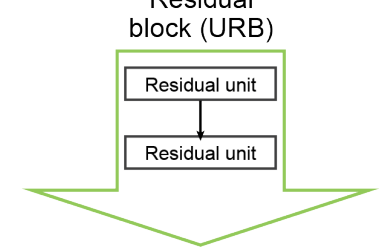

723

724 Extended Data Fig. 15 | Neural network-based prediction of smFISH using brightfield z-stacks.

725 


\section{References}

728 1. Biancalani, T. et al. Deep learning and alignment of spatially-resolved whole transcriptomes of

729 single cells in the mouse brain with Tangram. bioRxiv 2020.08.29.272831 (2020)

$730 \quad$ doi:10.1101/2020.08.29.272831.

731 2. Germond, A., Panina, Y., Shiga, M., Niioka, H. \& Watanabe, T. M. Following Embryonic Stem

732 Cells, Their Differentiated Progeny, and Cell-State Changes During iPS Reprogramming by Raman

733 Spectroscopy. Anal. Chem. 92, 14915-14923 (2020). 